\title{
Structural Variations of 2D and 3D Lanthanide Oxalate Frameworks Hydrothermally Synthesized in the Presence of Hydrazinium Ions.
}

Marine Ellart, Florent Blanchard, Murielle Rivenet, Francis Abraham

1. Univ. Lille Nord de France, Unité de Catalyse et de Chimie du Solide, UCCS, UMR CNRS 8181, ENSCL-USTL, BP 90108, 59652 Villeneuve d'Ascq Cedex, France

Supplementary Information

Table S1. Type of phase obtained depending the lanthanide and the $\mathrm{pH}$.

\begin{tabular}{|l|l|l|l|l|l|l|l|l|l|l|l|l|l|l|}
\hline $\mathbf{L n}$ & La & Ce & Pr & Nd & Sm & Eu & Gd & Tb & Dy & Ho & Er & Tm & Yb & Lu \\
\hline 1 & $1^{*}$ & $2 *$ & 2 & 2 & $2 *$ & 3 & 3 & 3 & $3 *$ & 3 & 4 & 4 & 5 & 6 \\
\hline 3 & 1 & $2 *$ & 2 & 2 & 3 & 3 & 3 & 3 & 3 & 3 & 4 & 4 & 5 & $5 *$ \\
\hline
\end{tabular}

Note. * Weak reflections are observed in addition to the reflections of the phase of interest and correspond to unidentified phases 
Table S2. Characteristics of the hydrogen bonds involving hydrazinium ions in $\mathrm{N}_{2} \mathrm{H}_{5}\left[\mathrm{La}\left(\mathrm{C}_{2} \mathrm{O}_{4}\right)_{2}\right]$ (1), water molecules and hydrazinium ions in $\mathrm{N}_{2} \mathrm{H}_{5}\left[\left\{\mathrm{Ho}\left(\mathrm{H}_{2} \mathrm{O}\right)\right\}\left(\mathrm{C}_{2} \mathrm{O}_{4}\right)_{2}\right](\mathbf{3 b})$ and water molecules in $\left[\left\{\mathrm{Er}\left(\mathrm{H}_{2} \mathrm{O}\right)\right\}_{2}\left(\mathrm{C}_{2} \mathrm{O}_{4}\right)_{3}\right] \cdot \mathrm{H}_{2} \mathrm{O}(4)$ and (6). Distances in $\AA$, angles in ${ }^{\circ}$.

\begin{tabular}{|c|c|c|c|c|c|}
\hline & & $\begin{array}{c}\mathrm{N}-\mathrm{O} \\
\text { or } \mathrm{O}-\mathrm{O}\end{array}$ & $\begin{array}{c}\mathrm{N}-\mathrm{H} \\
\text { or } \mathrm{O}-\mathrm{H}\end{array}$ & $\mathrm{H}---\mathrm{O}$ & $\begin{array}{c}\mathrm{N}-\mathrm{H}-\mathrm{-}-\mathrm{O} \\
\text { or } \\
\mathrm{O}-\mathrm{H}---\mathrm{O}\end{array}$ \\
\hline \multirow{5}{*}{$(1-\mathbf{L a})$} & $\mathrm{N} 1$ - H1 - - O & $2.818(2)$ & $0.96(2)$ & $1.92(2)$ & $154(2)$ \\
\hline & $\mathrm{N} 1-\mathrm{H} 2--\mathrm{O} 5$ & $2.845(2)$ & $0.90(3)$ & $2.02(2)$ & $152(2)$ \\
\hline & $\mathrm{N} 1-\mathrm{H} 3$ - - - O4 & $2.939(2)$ & $0.94(2)$ & $2.06(2)$ & $155(2)$ \\
\hline & $\mathrm{N} 2-\mathrm{H} 4---\mathrm{O} 8$ & $3.068(3)$ & $0.99(3)$ & $2.21(3)$ & $144(2)$ \\
\hline & $\mathrm{N} 2-\mathrm{H} 5---\mathrm{O} 6$ & $2.977(2)$ & $0.95(3)$ & $2.09(3)$ & $155(2)$ \\
\hline \multirow{5}{*}{ (3-Tb) } & Ow1 - Hw1A - - O1 & $2.836(6)$ & $0.81(6)$ & $2.16(6)$ & $140(5)$ \\
\hline & Ow1 - Hw1B - - O7 & $2.774(6)$ & $0.81(6)$ & $1.98(6)$ & $164(6)$ \\
\hline & $\mathrm{N} 1-\mathrm{H} 1---\mathrm{O} 6$ & $2.868(7)$ & $0.86(5)$ & $2.02(5)$ & $166(5)$ \\
\hline & $\mathrm{N} 1-\mathrm{H} 2---\mathrm{O} 8$ & $2.820(7)$ & $0.89(5)$ & $1.94(5)$ & $168(5)$ \\
\hline & $\mathrm{N} 1-\mathrm{H} 3---\mathrm{O} 3$ & $2.829(7)$ & $0.89(4)$ & $1.96(4)$ & $167(4)$ \\
\hline \multirow{5}{*}{ (3-Ho) } & Ow1 - Hw1A - - O1 & $2.839(4)$ & $0.81(4)$ & $2.13(4)$ & $145(3)$ \\
\hline & Ow1 - Hw1B - - O7 & $2.768(4)$ & $0.82(4)$ & $1.97(4)$ & $164(3)$ \\
\hline & $\mathrm{N} 1-\mathrm{H} 1--\mathrm{O} 6$ & $2.831(4)$ & $0.88(3)$ & $1.96(3)$ & $174(3)$ \\
\hline & $\mathrm{N} 1-\mathrm{H} 2--\mathrm{O} 8$ & $2.788(4)$ & $0.88(3)$ & $1.91(3)$ & $175(4)$ \\
\hline & $\mathrm{N} 1-\mathrm{H} 3---\mathrm{O} 3$ & $2.790(4)$ & $0.89(3)$ & $1.92(3)$ & $166(2)$ \\
\hline \multirow{9}{*}{ (4-Er) } & Ow1 - H1 - - O11 & $2.751(3)$ & $0.78(6)$ & $1.98(6)$ & $169(5)$ \\
\hline & Ow1 - H2 - - Ow2 & $2.768(3)$ & $0.90(4)$ & $1.89(4)$ & $163(5)$ \\
\hline & $\mathrm{Ow} 2-\mathrm{H} 3--$ - O4 & $2.731(3)$ & $0.76(5)$ & $1.98(5)$ & 171(4) \\
\hline & Ow2 - H4 - - Ow3 & $2.628(7)$ & $0.81(4)$ & $1.82(4)$ & $171(5)$ \\
\hline & Ow2 - H4 - - Ow3 & $2.624(5)$ & $0.81(4)$ & $1.84(5)$ & $160(5)$ \\
\hline & Ow3 - $\mathrm{H}^{1}-$ - O9 & $2.791(7)$ & & & \\
\hline & Ow3 $-\mathrm{H}^{1}---\mathrm{O} 2$ & $2.829(7)$ & & & \\
\hline & Ow3' - $\mathrm{H}^{1}-$ - O9 & $2.798(5)$ & & & \\
\hline & $\mathrm{Ow}^{\prime}-\mathrm{H}^{1}---\mathrm{O} 2$ & $2.779(6)$ & & & \\
\hline \multirow{6}{*}{ (6-Lu) } & Ow1 - H1 - - O2 & $2.92(2)$ & $0.83(3)$ & $2.09(3)$ & $170(2)$ \\
\hline & Ow1 - H2 - - Ow3 & $2.73(2)$ & $0.84(2)$ & $1.90(2)$ & $173(2)$ \\
\hline & $\mathrm{Ow} 2-\mathrm{H} 3---\mathrm{O} 2$ & $2.96(2)$ & $0.84(3)$ & $2.13(3)$ & 171(3) \\
\hline & $\mathrm{Ow} 2-\mathrm{H} 4---\mathrm{O} 6$ & $2.88(1)$ & $0.84(3)$ & $2.04(3)$ & $179(2)$ \\
\hline & Ow3 - H5 - - Ow2 & $3.32(3)$ & $0.85(4)$ & $2.56(5)$ & $148(4)$ \\
\hline & Ow3 - H6 - - O5 & $3.104(7)$ & $0.85(5)$ & $2.28(5)$ & $163(4)$ \\
\hline
\end{tabular}

Note 1. Ow3 is disordered on two sites Ow3 and Ow3' and the H atoms not localized. 
Table S3. Unit cell parameters refined from X-ray powder patterns for the different lanthanide oxalates.

\begin{tabular}{|l|l|l|l|l|l|l|l|l|}
\hline Type & Ln & $\mathrm{a}(\AA)$ & $\mathrm{b}(\AA)$ & $\mathrm{c}(\AA)$ & $\alpha\left(^{\circ}\right)$ & $\beta\left(^{\circ}\right)$ & $\gamma\left({ }^{\circ}\right)$ & $\mathrm{V}\left(\AA^{3}\right)$ \\
\hline 1 & $\mathrm{La}$ & $6.05995(12)$ & $15.6175(3)$ & $8.95093(17)$ & 90 & $95.2919(10)$ & 90 & $843.51(3)$ \\
\hline \multirow{4}{*}{2} & $\mathrm{Ce}$ & $16.3758(7)$ & $12.3015(5)$ & $11.5598(4)$ & 90 & $116.7502(18)$ & 90 & $2079.47(14)$ \\
\cline { 2 - 9 } & $\mathrm{Pr}$ & $16.3537(9)$ & $12.2221(6)$ & $11.4989(5)$ & 90 & $116.699(2)$ & 90 & $2053.32(18)$ \\
\cline { 2 - 9 } & $\mathrm{Nd}$ & $16.3353(10)$ & $12.1575(7)$ & $11.4477(6)$ & 90 & $116.651(2)$ & 90 & $2031.9(2)$ \\
\cline { 2 - 9 } & $\mathrm{Sm}$ & $16.3047(11)$ & $12.0329(8)$ & $11.3608(7)$ & 90 & $116.560(2)$ & 90 & $1993.7(2)$ \\
\hline \multirow{5}{*}{3} & $\mathrm{Sm}$ & $12.5997(3)$ & $12.1724(4)$ & $12.8165(3)$ & 90 & 90 & 90 & $1965.66(9)$ \\
\cline { 2 - 9 } & $\mathrm{Eu}$ & $12.6040(7)$ & $12.1207(7)$ & $12.7775(7)$ & 90 & 90 & 90 & $1952.00(18)$ \\
\cline { 2 - 9 } & $\mathrm{Gd}$ & $12.6046(8)$ & $12.0729(9)$ & $12.7434(9)$ & 90 & 90 & 90 & $1939.2(2)$ \\
\cline { 2 - 9 } & $\mathrm{Tb}$ & $12.6123(7)$ & $12.0103(8)$ & $12.6938(8)$ & 90 & 90 & 90 & $1922.8(2)$ \\
\cline { 2 - 9 } & $\mathrm{Dy}$ & $12.6261(8)$ & $11.9701(9)$ & $12.6387(9)$ & 90 & 90 & 90 & $1910.2(2)$ \\
\cline { 2 - 9 } & $\mathrm{Ho}$ & $12.6194(8)$ & $11.9097(7)$ & $12.6216(8)$ & 90 & 90 & 90 & $1896.9(2)$ \\
\hline \multirow{3}{*}{4} & $\mathrm{Er}$ & $10.5350(2)$ & $11.5952(3)$ & $11.6061(3)$ & 90 & $115.0627(8)$ & 90 & $1284.27(6)$ \\
\cline { 2 - 9 } & $\mathrm{Tm}$ & $10.5151(3)$ & $11.5416(4)$ & $11.5847(3)$ & 90 & $115.0532(7)$ & 90 & $1273.65(6)$ \\
\hline 5 & $\mathrm{Yb}$ & $9.1948(9)$ & $15.4813(14)$ & $32.133(3)$ & 90 & 90 & 90 & $4574.1(7)$ \\
\cline { 2 - 9 } & $\mathrm{Lu}$ & $9.1703(4)$ & $15.4952(6)$ & $32.0309(14)$ & 90 & 90 & 90 & $4551.5(3)$ \\
\hline 6 & $\mathrm{Lu}$ & $6.2585(3)$ & $6.6324(3)$ & $9.6004(6)$ & $75.082(4)$ & $80.548(5)$ & $81.843(5)$ & $377.76(4)$ \\
\hline
\end{tabular}

Table S4. Density and total loss of mass for the different lanthanide oxalates

\begin{tabular}{|l|l|l|l|l|l|}
\hline \multirow{2}{*}{ Type } & \multirow{2}{*}{ Ln } & \multicolumn{3}{|c|}{ Density } & \multicolumn{2}{c|}{ TGA } \\
\cline { 3 - 6 } & & $\rho_{\text {calc }} /{\mathrm{g} . \mathrm{cm}^{-3}}^{-3}$ & $\rho_{\text {mes. }} / \mathrm{g} . c m^{-3}$ & $\Delta \mathrm{m}_{\text {theo }} . \%$ & $\Delta \mathrm{m}_{\text {exp }} . \%$ \\
\hline 1 & $\mathrm{La}$ & 2.740 & $2.764(3)$ & 53.2 & 53.6 \\
\hline \multirow{5}{*}{3} & $\mathrm{Pr}$ & 2.520 & $2.589(5)$ & 55.9 & 54.8 \\
\cline { 2 - 6 } & $\mathrm{Nd}$ & 2.550 & $2.556(6)$ & 56.8 & 56.6 \\
\hline \multirow{5}{*}{3} & $\mathrm{Sm}$ & 2.550 & $2.587(4)$ & 53.8 & 52.5 \\
\cline { 2 - 6 } & $\mathrm{Eu}$ & 2.570 & $2.574(7)$ & 53.6 & 52.9 \\
\cline { 2 - 6 } & $\mathrm{Gd}$ & 2.628 & $2.638(4)$ & 52.9 & 52.3 \\
\cline { 2 - 6 } & $\mathrm{Tb}$ & 2.662 & $2.666(4)$ & 52.6 & 50.6 \\
\cline { 2 - 6 } & $\mathrm{Dy}$ & 2.706 & $2.741(2)$ & 52.1 & 51.9 \\
\cline { 2 - 6 } & $\mathrm{Ho}$ & 2.734 & $2.777(4)$ & 51.8 & 52.0 \\
\hline \multirow{3}{*}{4} & $\mathrm{Er}$ & 3.372 & $3.288(7)$ & 41.4 & 41.0 \\
\cline { 2 - 6 } & $\mathrm{Tm}$ & 3.326 & $3.339(6)$ & 41.3 & 40.8 \\
\hline 5 & $\mathrm{Yb}$ & 2.532 & $2,538(5)$ & 54.8 & 54.3 \\
\hline 6 & $\mathrm{Lu}$ & 3.174 & $3.162(5)$ & 45.0 & 47.5 \\
\hline
\end{tabular}


Table S5. The principal IR bands of oxalate and hydrazinium ions in the different lanthanide oxalates with tentative assignment.

\begin{tabular}{|c|c|c|c|c|c|c|c|c|c|c|c|c|c|c|c|}
\hline & & $\begin{array}{l}\text { (1) } \\
\mathrm{La}\end{array}$ & $\begin{array}{l}\text { (2) } \\
\mathrm{Ce}\end{array}$ & $\begin{array}{l}\text { (2) } \\
\operatorname{Pr}\end{array}$ & $\begin{array}{l}\text { (2) } \\
\mathrm{Nd}\end{array}$ & $\begin{array}{l}\text { (3) } \\
\text { Sm }\end{array}$ & $\begin{array}{l}\text { (3) } \\
\mathrm{Eu}\end{array}$ & $\begin{array}{l}\text { (3) } \\
\text { Gd }\end{array}$ & $\begin{array}{l}\text { (3) } \\
\mathrm{Tb}\end{array}$ & $\begin{array}{l}\text { (3) } \\
\text { Dy }\end{array}$ & $\begin{array}{l}\text { (3) } \\
\text { Ho }\end{array}$ & $\begin{array}{l}\text { (4) } \\
\text { Er }\end{array}$ & $\begin{array}{l}\text { (4) } \\
\text { Tm }\end{array}$ & $\begin{array}{l}\text { (5) } \\
\mathrm{Yb}\end{array}$ & $\begin{array}{l}\text { (6) } \\
\mathrm{Lu}\end{array}$ \\
\hline \multirow[t]{6}{*}{$\left(\mathrm{C}_{2} \mathrm{O}_{4}\right)^{2}$} & $v_{\text {as }} \mathrm{CO}$ & 1617 & 1602 & 1604 & 1609 & 1611 & 1613 & 1622 & 1621 & 1616 & 1622 & 1619 & 1628 & 1620 & 1622 \\
\hline & $v_{\mathrm{s}} \mathrm{CO}$ & 1468 & 1458 & 1459 & 1462 & 1461 & 1485 & 1491 & 1506 & 1486 & 1505 & 1480 & 1488 & & \\
\hline & $\begin{array}{c}v \mathrm{CO}+ \\
v \mathrm{CC}\end{array}$ & 1375 & 1358 & 1354 & 1363 & 1364 & 1357 & 1355 & 1359 & 1357 & 1360 & 1348 & 1353 & 1363 & 1360 \\
\hline & $\begin{array}{l}v \mathrm{CO}+ \\
\delta \text { OCO }\end{array}$ & 1311 & 1305 & 1304 & 1308 & 1308 & 1315 & 1316 & 1313 & 1310 & 1316 & 1289 & 1297 & 1323 & 1323 \\
\hline & $v \mathrm{CC}$ & 908 & 904 & 903 & 900 & 858 & 858 & 857 & 857 & 857 & 857 & 857 & 877 & & 890 \\
\hline & $\begin{array}{l}\text { Asym. } \\
\delta \text { OCO }\end{array}$ & 786 & 785 & 785 & 788 & 787 & 791 & 792 & 793 & 792 & 793 & 792 & 790 & 801 & 800 \\
\hline \multirow[t]{6}{*}{$\mathrm{N}_{2} \mathrm{H}_{5}{ }^{+}$} & $v \mathrm{NH}_{2}$ & 3315 & 3313 & 3310 & 3325 & 3356 & 3356 & 3358 & 3360 & 3364 & 3410 & & & 3390 & \\
\hline & $\delta \mathrm{NH}_{2}$ & 1589 & & & & 1550 & 1548 & 1549 & 1573 & 1550 & 1573 & & & 1526 & \\
\hline & $\mathrm{T} \mathrm{NH}_{2}$ & & 1161 & 1162 & 1166 & 1141 & 1139 & 1144 & 1145 & 1140 & 1140 & & & & \\
\hline & $\delta \mathrm{HNH}$ & 1100 & 1104 & 1103 & 1107 & 1106 & 1093 & 1089 & 1094 & 1091 & 1093 & & & 1094 & \\
\hline & $v \mathrm{NN}^{1}$ & & 991 & 995 & 996 & & & & & & & & & & \\
\hline & $v \mathrm{NN}^{2}$ & 956 & 957 & 958 & 956 & 932 & 933 & 935 & 935 & 936 & 937 & & & 960 & \\
\hline
\end{tabular}

Notes. ${ }^{1}$ Coordinated $\mathrm{N}_{2} \mathrm{H}_{5}{ }^{+}$ion; ${ }^{2}$ uncoordinated $\mathrm{N}_{2} \mathrm{H}_{5}{ }^{+}$ion 
Figure S1. Photos and SEM images of 1-La, 2-Ce, 2-Pr, 2-Nd, 3-Sm, 3-Eu, 3-Tb, 3-Dy, 3-Ho, 4-Er, 4-Tm and 5-Yb lanthanide oxalates crystals.
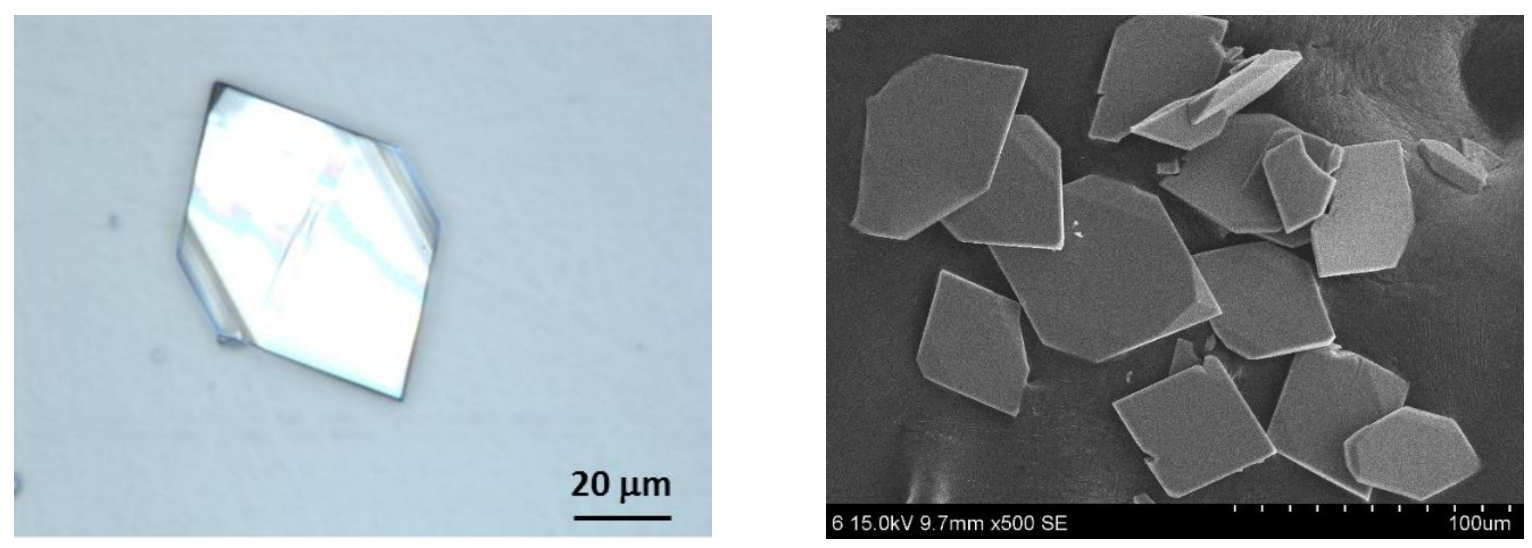

Compound $1\left(\mathrm{~N}_{2} \mathrm{H}_{5}\right) \mathrm{La}\left(\mathrm{C}_{2} \mathrm{O}_{4}\right)_{2}$
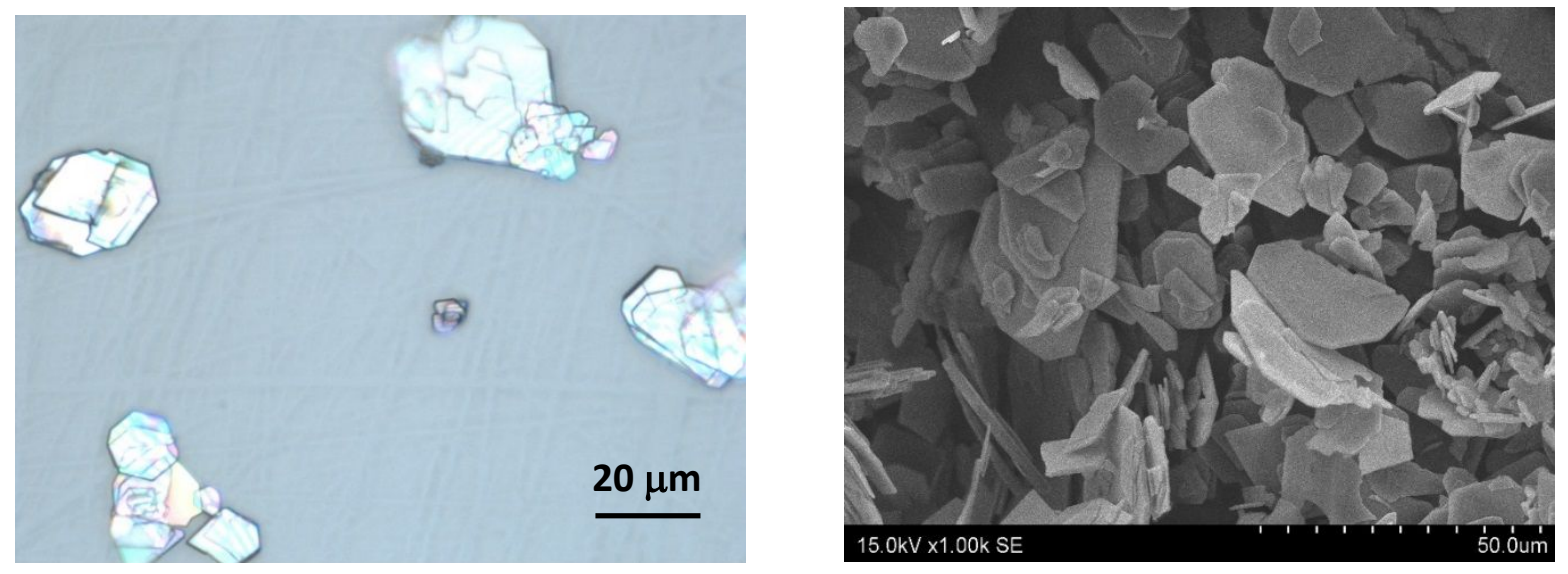

Compound 2-Ce $\mathrm{N}_{2} \mathrm{H}_{5}\left[\left\{\mathrm{Ce}_{2}\left(\mathrm{~N}_{2} \mathrm{H}_{5}\right)\right\}\left(\mathrm{C}_{2} \mathrm{O}_{4}\right)_{4}\right] \cdot 4 \mathrm{H}_{2} \mathrm{O}$
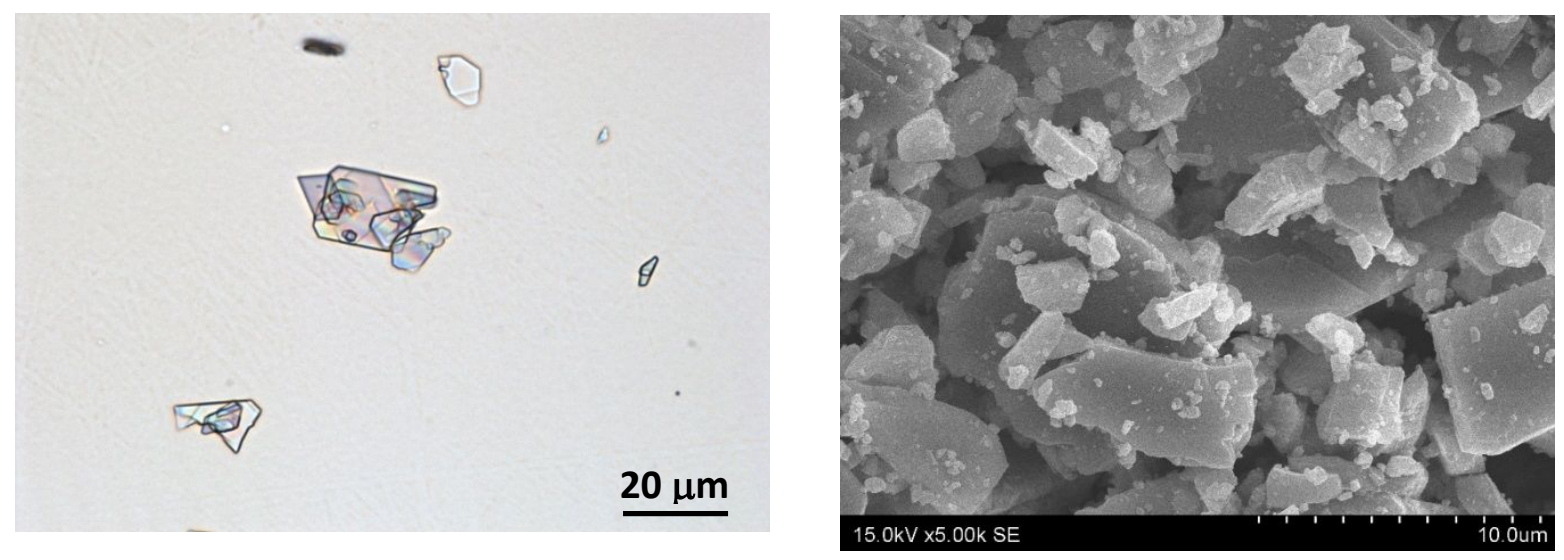

Compound 2-Pr $\mathrm{N}_{2} \mathrm{H}_{5}\left[\left\{\mathrm{Pr}_{2}\left(\mathrm{~N}_{2} \mathrm{H}_{5}\right)\right\}\left(\mathrm{C}_{2} \mathrm{O}_{4}\right)_{4}\right] \cdot 4 \mathrm{H}_{2} \mathrm{O}$ 

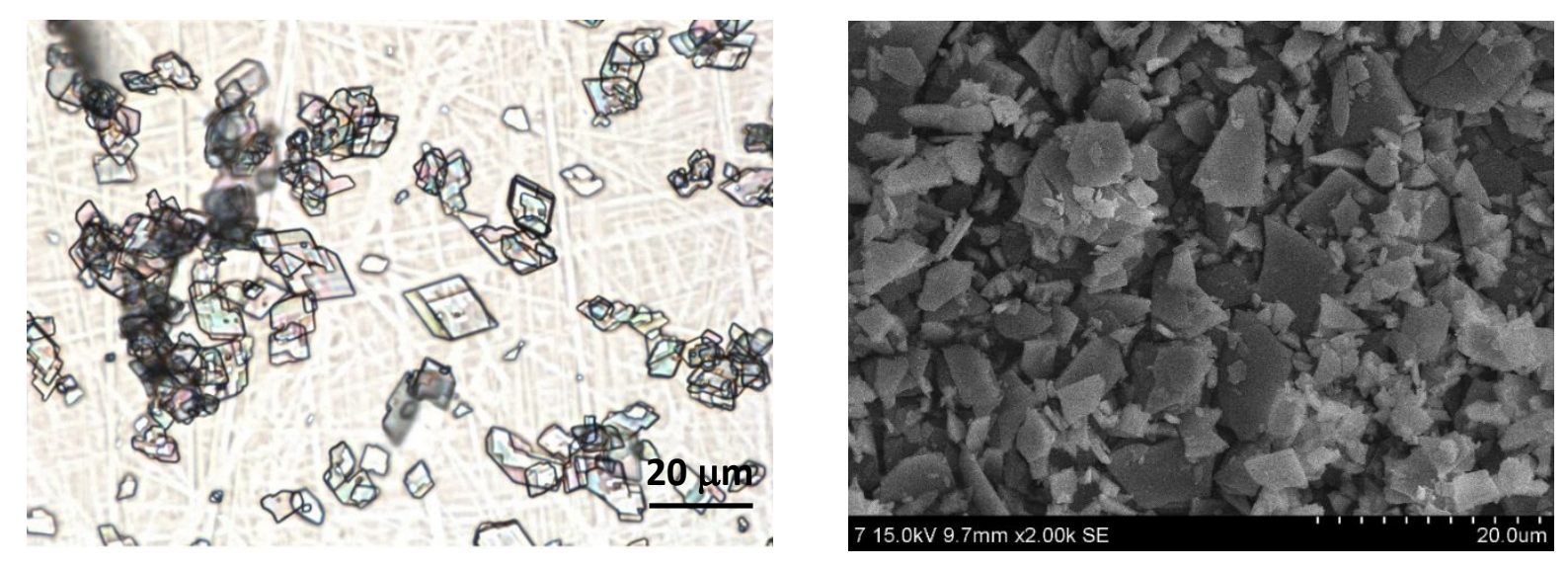

Compound 2-Nd $\mathrm{N}_{2} \mathrm{H}_{5}\left[\left\{\mathrm{Nd}_{2}\left(\mathrm{~N}_{2} \mathrm{H}_{5}\right)\right\}\left(\mathrm{C}_{2} \mathrm{O}_{4}\right)_{4}\right] \cdot 4 \mathrm{H}_{2} \mathrm{O}$
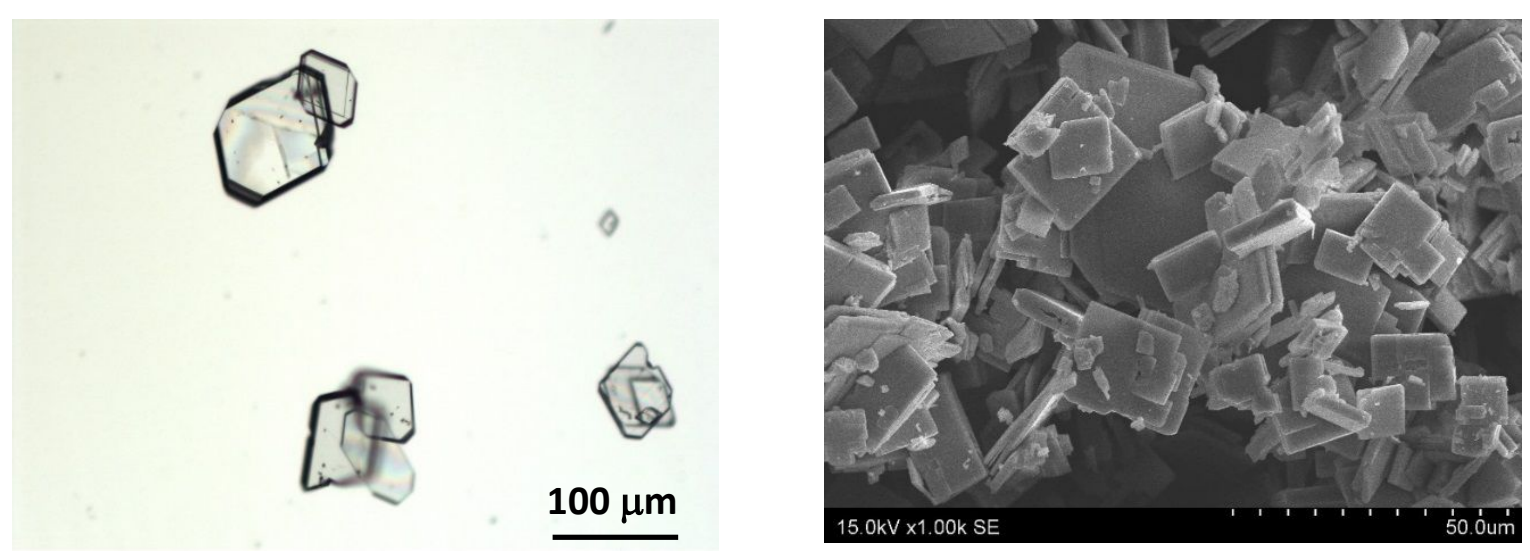

Compound 3-Sm $\left(\mathrm{N}_{2} \mathrm{H}_{5}\right) \mathrm{Sm}\left(\mathrm{C}_{2} \mathrm{O}_{4}\right)_{2} \cdot \mathrm{H}_{2} \mathrm{O}$
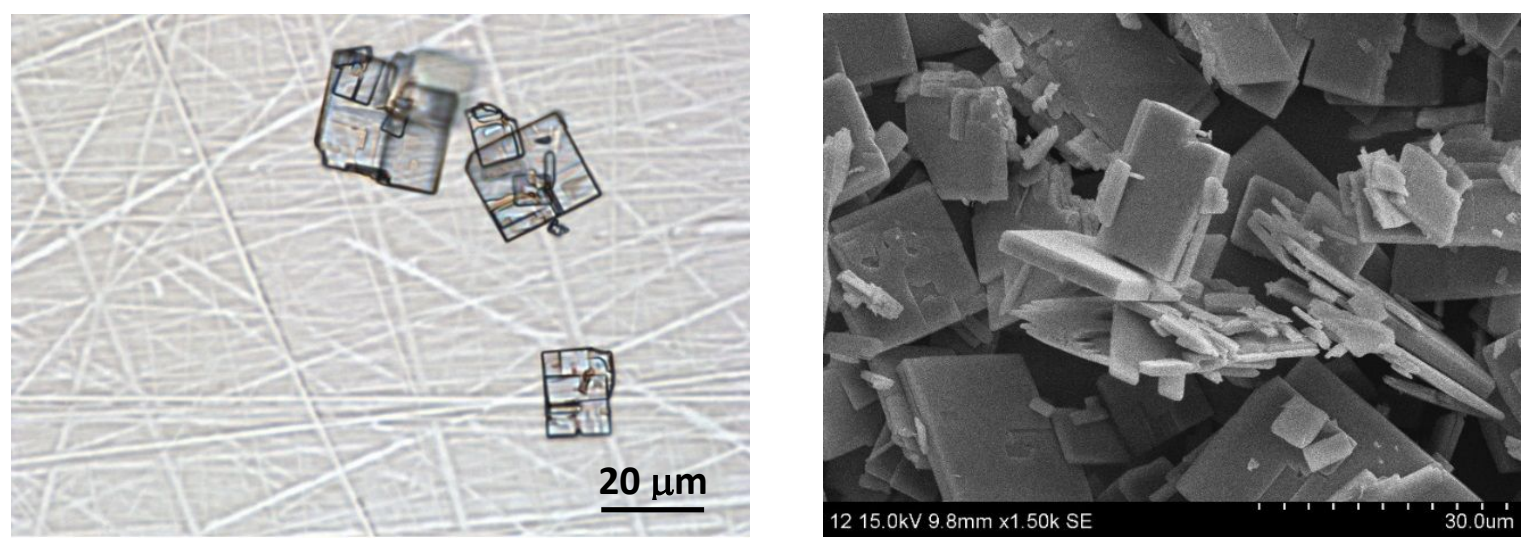

Compound 3-Eu $\left(\mathrm{N}_{2} \mathrm{H}_{5}\right) \mathrm{Eu}\left(\mathrm{C}_{2} \mathrm{O}_{4}\right)_{2} \cdot \mathrm{H}_{2} \mathrm{O}$ 

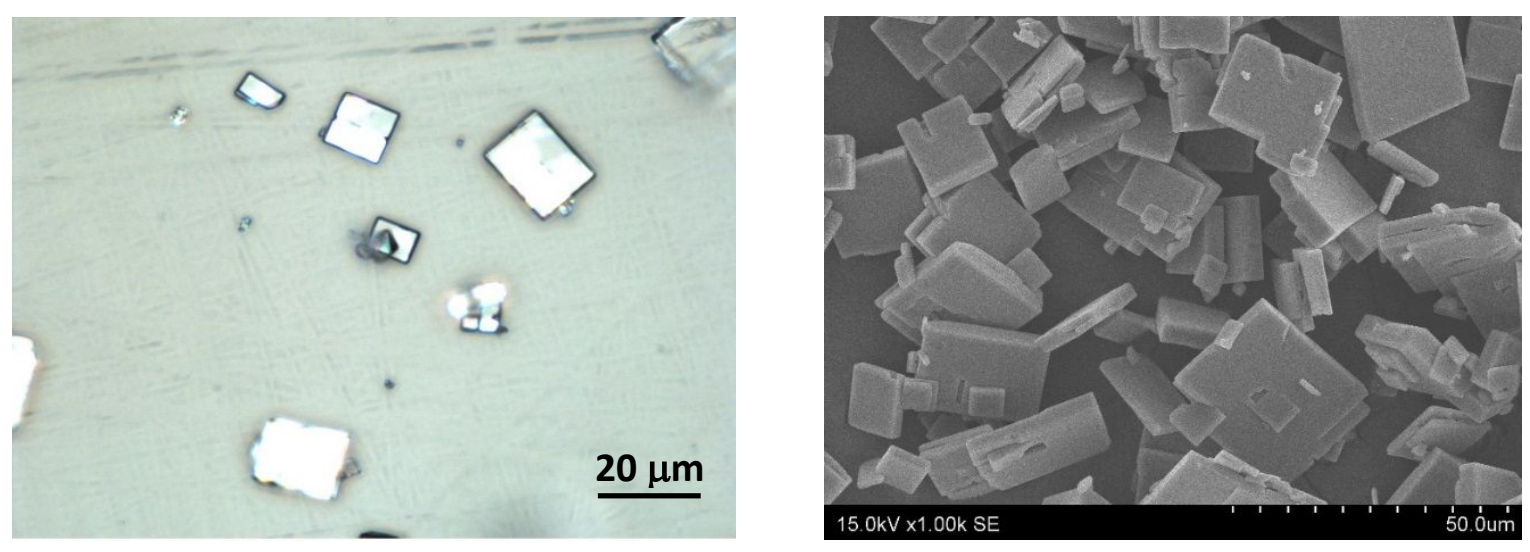

Compound 3-Tb $\left(\mathrm{N}_{2} \mathrm{H}_{5}\right) \mathrm{Tb}\left(\mathrm{C}_{2} \mathrm{O}_{4}\right)_{2} \cdot \mathrm{H}_{2} \mathrm{O}$
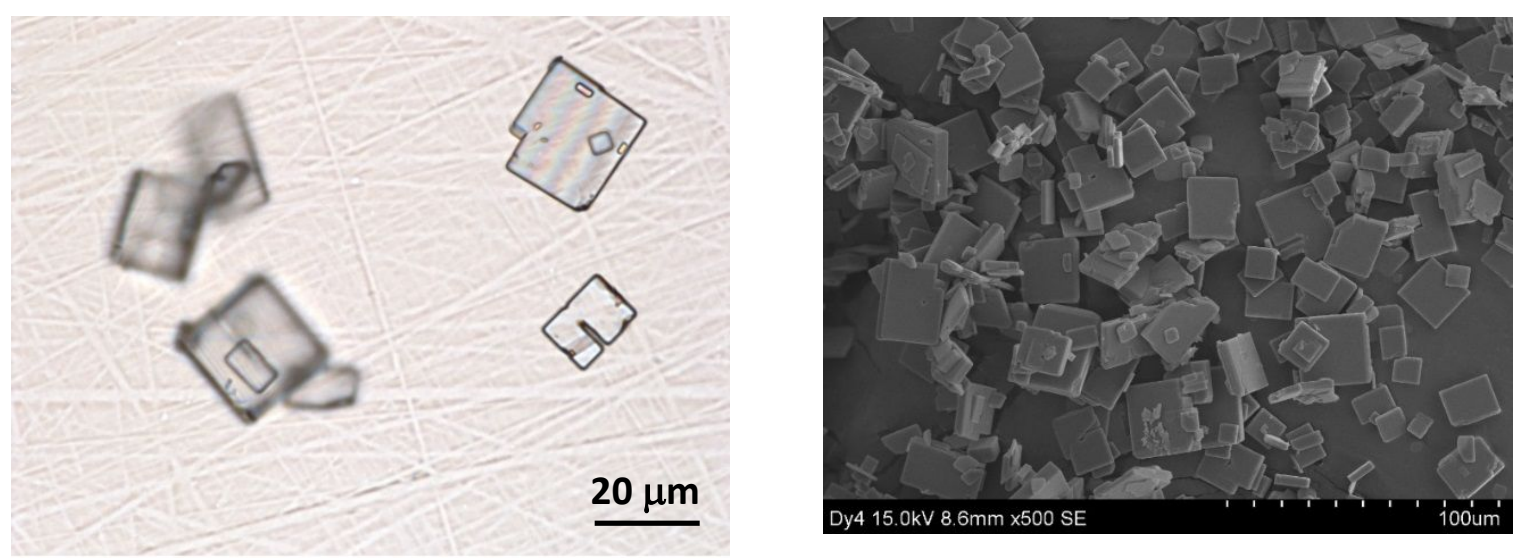

Compound 3-Dy $\left(\mathrm{N}_{2} \mathrm{H}_{5}\right) \mathrm{Dy}\left(\mathrm{C}_{2} \mathrm{O}_{4}\right)_{2} \cdot \mathrm{H}_{2} \mathrm{O}$
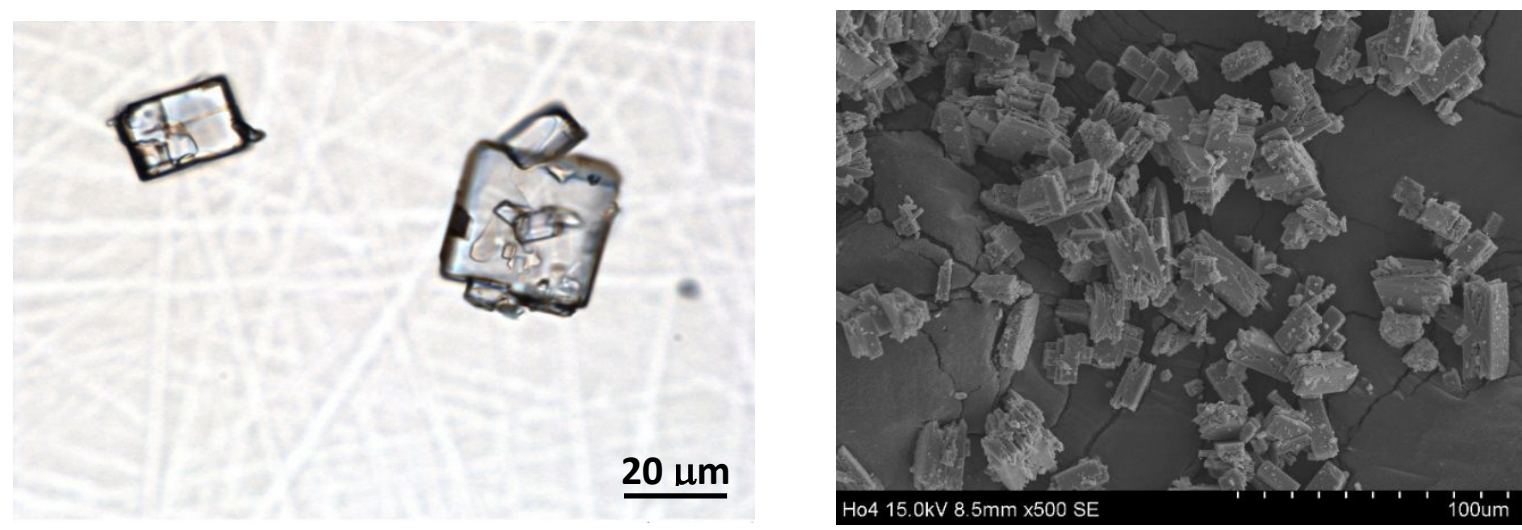

Compound 3-Ho $\left(\mathrm{N}_{2} \mathrm{H}_{5}\right) \mathrm{Ho}\left(\mathrm{C}_{2} \mathrm{O}_{4}\right)_{2} \cdot \mathrm{H}_{2} \mathrm{O}$ 

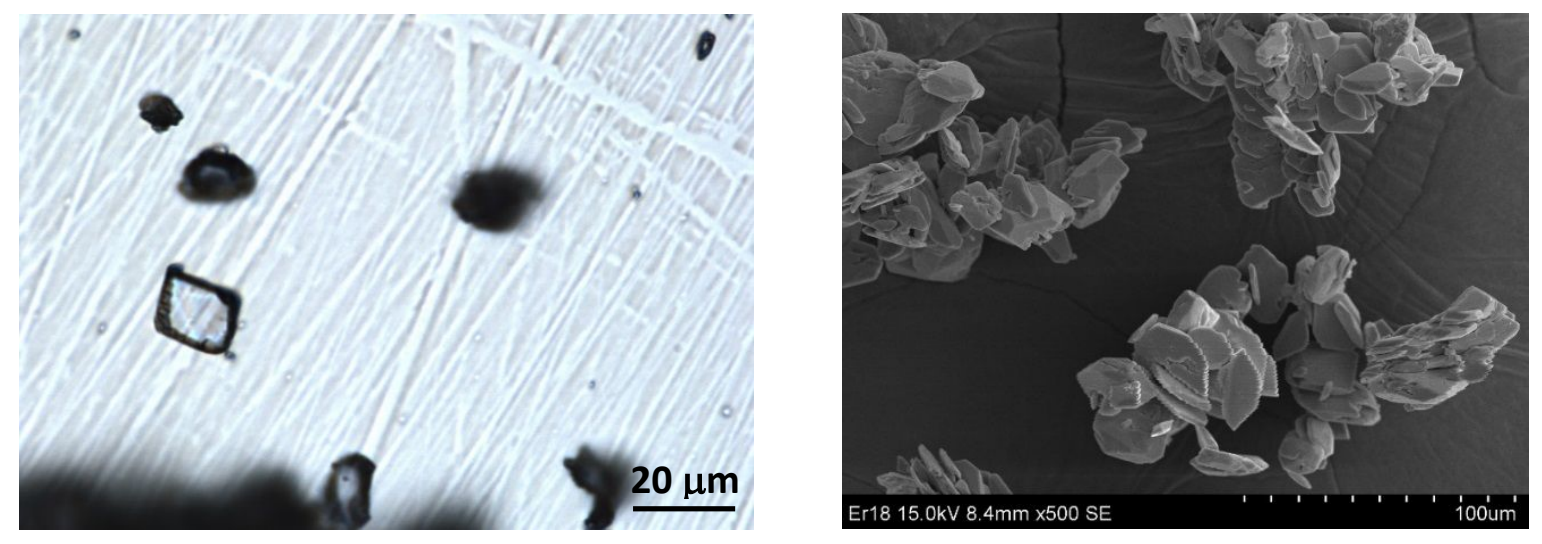

Compound 4-Er $\left[\left\{\operatorname{Er}\left(\mathrm{H}_{2} \mathrm{O}\right)\right\}_{2}\left(\mathrm{C}_{2} \mathrm{O}_{4}\right)_{3}\right] \cdot \mathrm{H}_{2} \mathrm{O}$
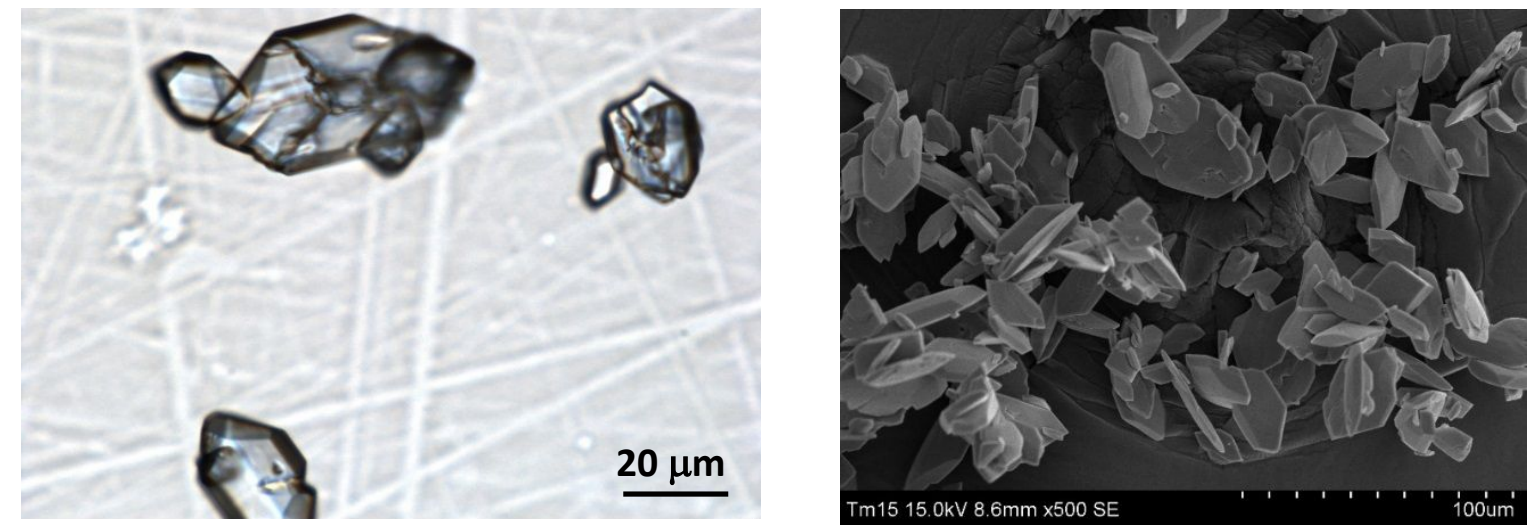

Compound 4-Tm $\left[\left\{\mathrm{Tm}\left(\mathrm{H}_{2} \mathrm{O}\right)\right\}_{2}\left(\mathrm{C}_{2} \mathrm{O}_{4}\right)_{3}\right] \cdot \mathrm{H}_{2} \mathrm{O}$
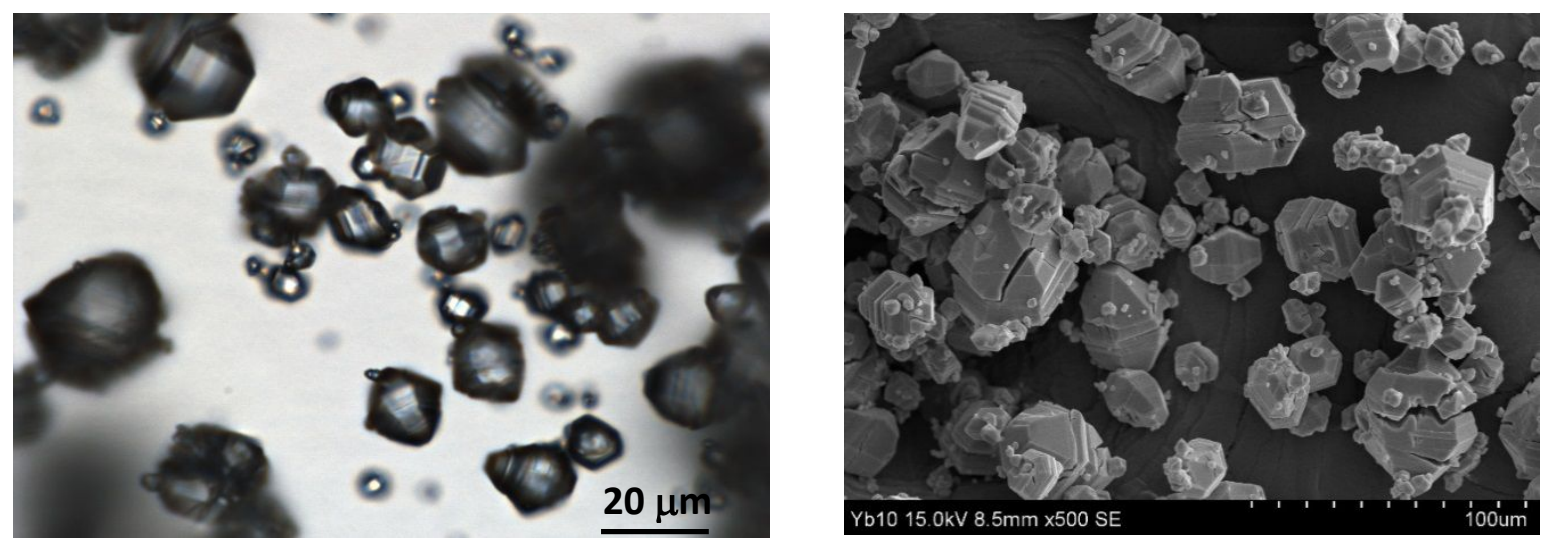

Compound 5- $\mathrm{Yb}\left(\mathrm{N}_{2} \mathrm{H}_{5}\right) \mathrm{Yb}\left(\mathrm{C}_{2} \mathrm{O}_{4}\right)_{2} \cdot 3 \mathrm{H}_{2} \mathrm{O}$ 
Figure S2. TGA analyses in air of 1-La, 2-Ce, 2-Pr, 2-Nd, 3-Sm, 3-Eu, 3-Gd, 3Tb, 3-Dy, 3-Ho, 4-Er, 4-Tm, 5-Yb and 6-Lu lanthanide oxalates.

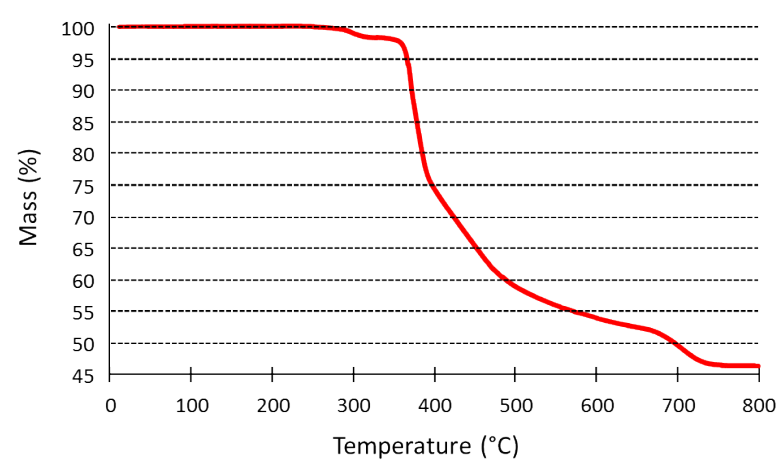

Compound 1

$\left(\mathrm{N}_{2} \mathrm{H}_{5}\right) \mathrm{La}\left(\mathrm{C}_{2} \mathrm{O}_{4}\right)_{2}$

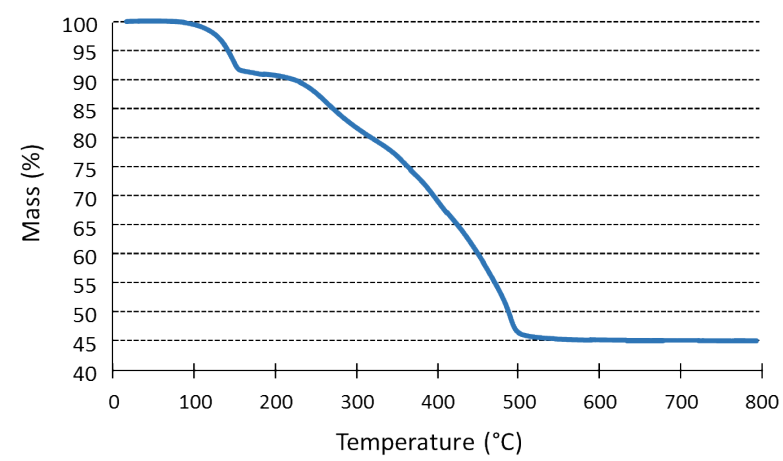

Compound 2-Pr

$\mathrm{N}_{2} \mathrm{H}_{5}\left[\left\{\mathrm{Pr}_{2}\left(\mathrm{~N}_{2} \mathrm{H}_{5}\right)\right\}\left(\mathrm{C}_{2} \mathrm{O}_{4}\right)_{4}\right] \cdot 4 \mathrm{H}_{2} \mathrm{O}$

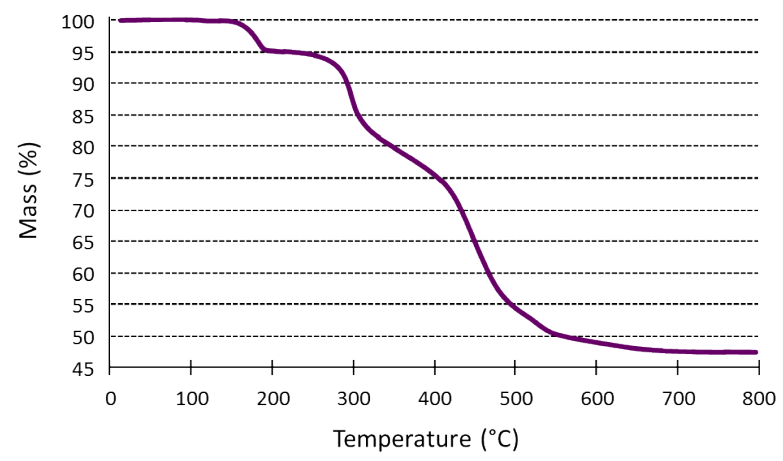

Compound 3-Sm $\left(\mathrm{N}_{2} \mathrm{H}_{5}\right) \mathrm{Sm}\left(\mathrm{C}_{2} \mathrm{O}_{4}\right)_{2} \cdot \mathrm{H}_{2} \mathrm{O}$

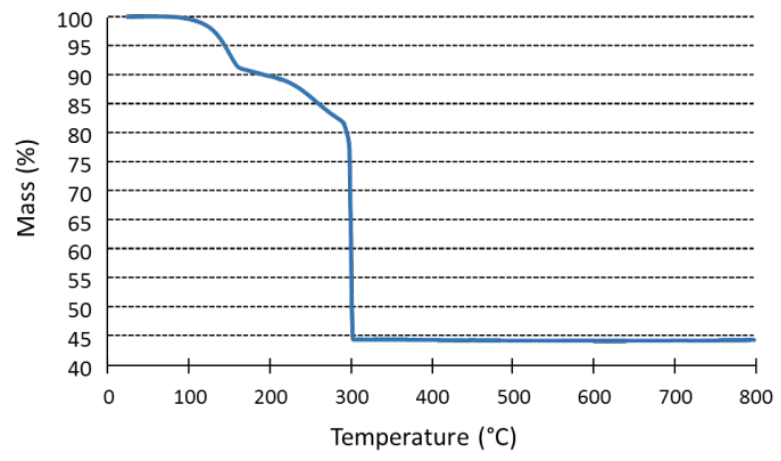

Compound 2-Ce

$\mathrm{N}_{2} \mathrm{H}_{5}\left[\left\{\mathrm{Ce}_{2}\left(\mathrm{~N}_{2} \mathrm{H}_{5}\right)\right\}\left(\mathrm{C}_{2} \mathrm{O}_{4}\right)_{4}\right] \cdot 4 \mathrm{H}_{2} \mathrm{O}$

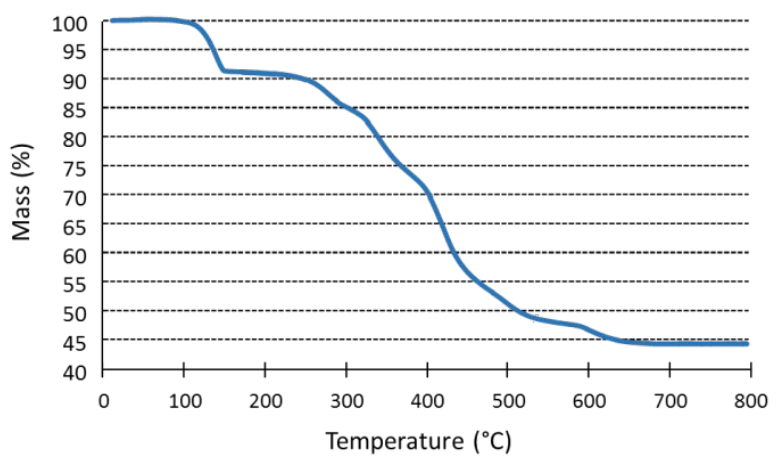

Compound 2-Nd

$\mathrm{N}_{2} \mathrm{H}_{5}\left[\left\{\mathrm{Nd}_{2}\left(\mathrm{~N}_{2} \mathrm{H}_{5}\right)\right\}\left(\mathrm{C}_{2} \mathrm{O}_{4}\right)_{4}\right] \cdot 4 \mathrm{H}_{2} \mathrm{O}$

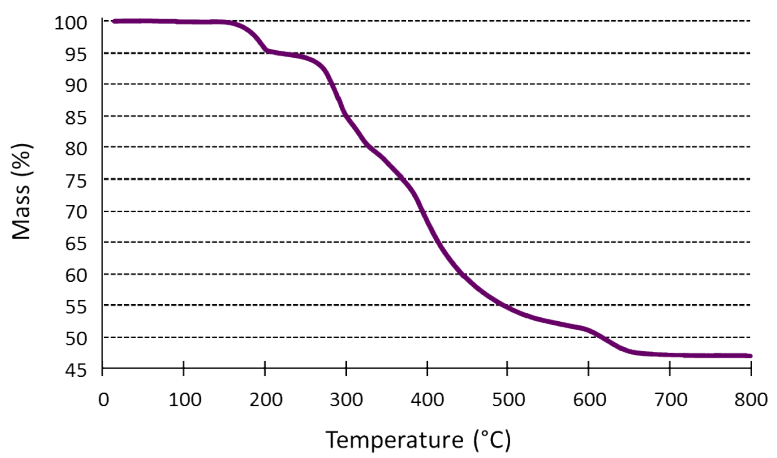

Compound 3-Eu $\left(\mathrm{N}_{2} \mathrm{H}_{5}\right) \mathrm{Eu}\left(\mathrm{C}_{2} \mathrm{O}_{4}\right)_{2} \cdot \mathrm{H}_{2} \mathrm{O}$ 


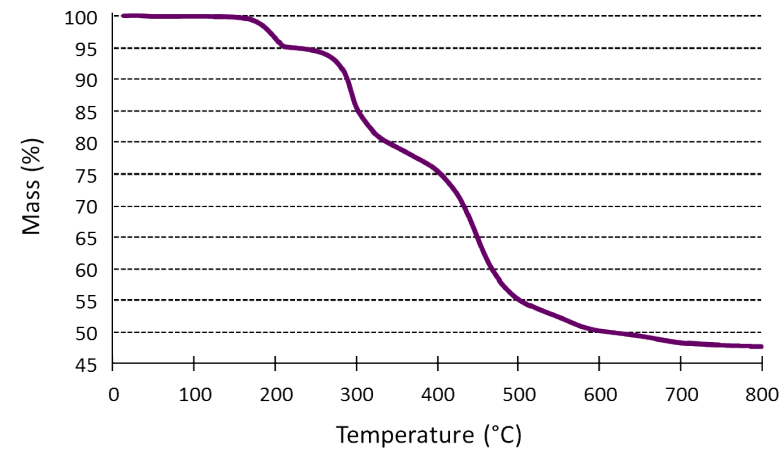

Compound 3-Gd $\left(\mathrm{N}_{2} \mathrm{H}_{5}\right) \mathrm{Gd}\left(\mathrm{C}_{2} \mathrm{O}_{4}\right)_{2} \cdot \mathrm{H}_{2} \mathrm{O}$

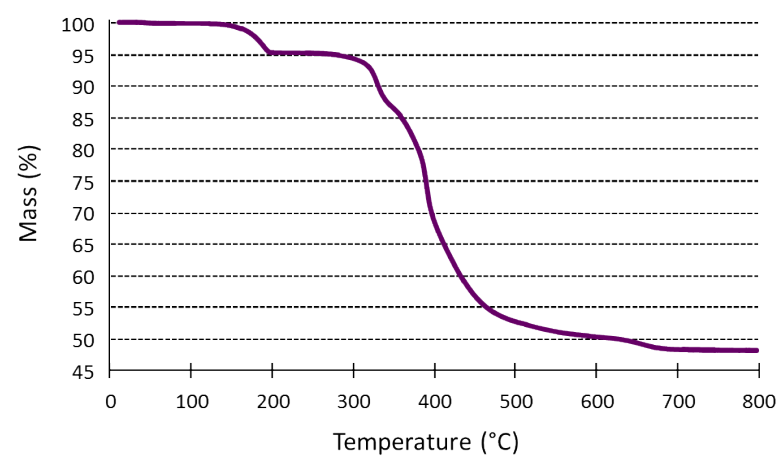

Compound 3-Dy $\left(\mathrm{N}_{2} \mathrm{H}_{5}\right) \mathrm{Dy}\left(\mathrm{C}_{2} \mathrm{O}_{4}\right)_{2} \cdot \mathrm{H}_{2} \mathrm{O}$

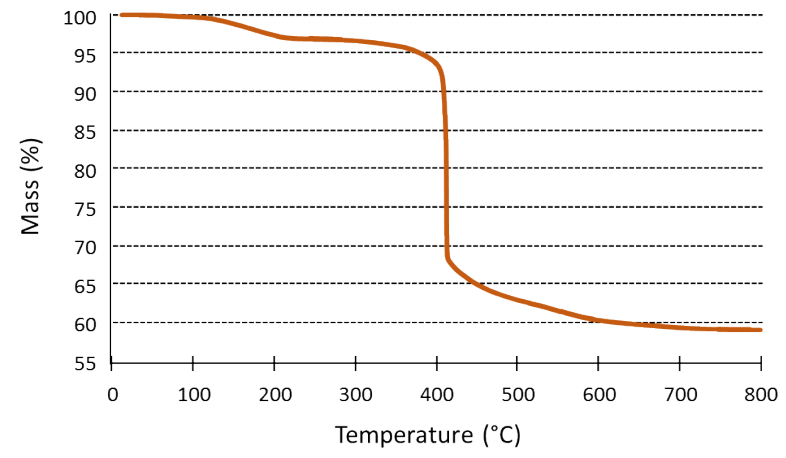

Compound 4-Er $\left[\left\{\operatorname{Er}\left(\mathrm{H}_{2} \mathrm{O}\right)\right\}_{2}\left(\mathrm{C}_{2} \mathrm{O}_{4}\right)_{3}\right] \cdot \mathrm{H}_{2} \mathrm{O}$

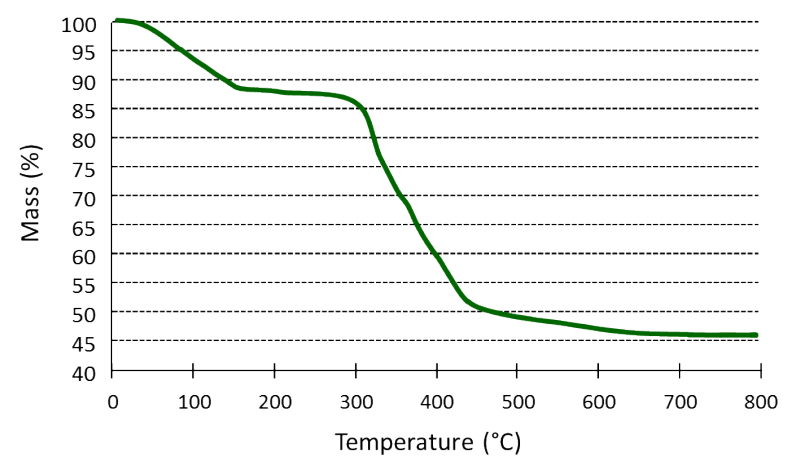

Compound 5- $\mathrm{Yb}\left(\mathrm{N}_{2} \mathrm{H}_{5}\right) \mathrm{Yb}\left(\mathrm{C}_{2} \mathrm{O}_{4}\right)_{2} .3 \mathrm{H}_{2} \mathrm{O}$

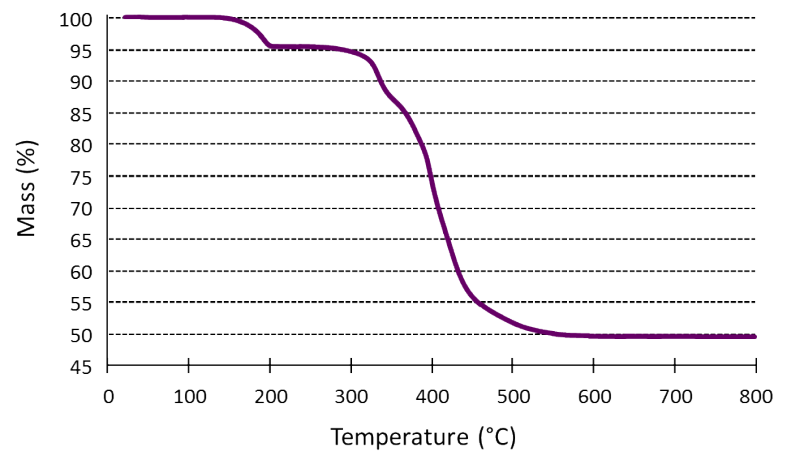

Compound 3-Tb $\left(\mathrm{N}_{2} \mathrm{H}_{5}\right) \mathrm{Tb}\left(\mathrm{C}_{2} \mathrm{O}_{4}\right)_{2} \cdot \mathrm{H}_{2} \mathrm{O}$

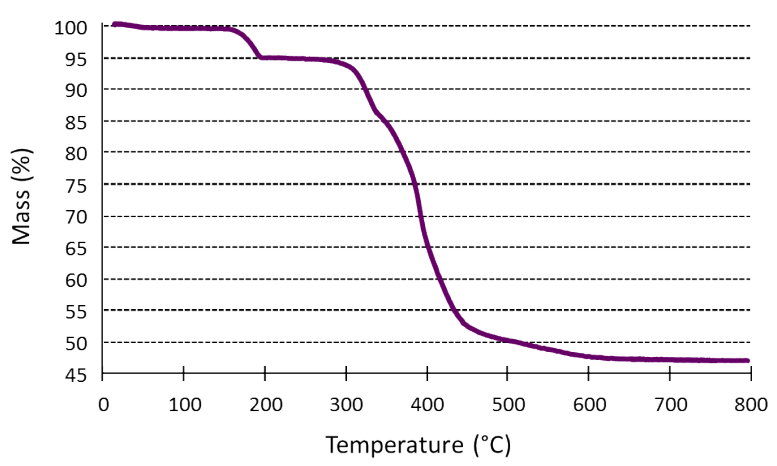

Compound 3-Ho $\left(\mathrm{N}_{2} \mathrm{H}_{5}\right) \mathrm{Ho}\left(\mathrm{C}_{2} \mathrm{O}_{4}\right)_{2} \cdot \mathrm{H}_{2} \mathrm{O}$

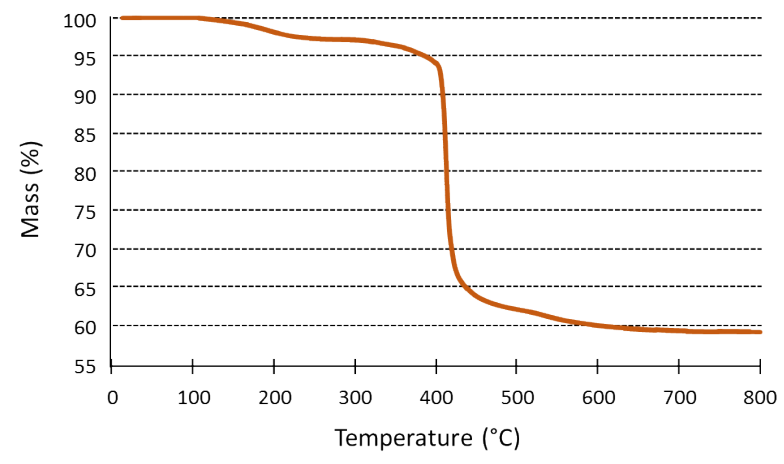

Compound 4-Tm $\left[\left\{\operatorname{Tm}\left(\mathrm{H}_{2} \mathrm{O}\right)\right\}_{2}\left(\mathrm{C}_{2} \mathrm{O}_{4}\right)_{3}\right] \cdot \mathrm{H}_{2} \mathrm{O}$

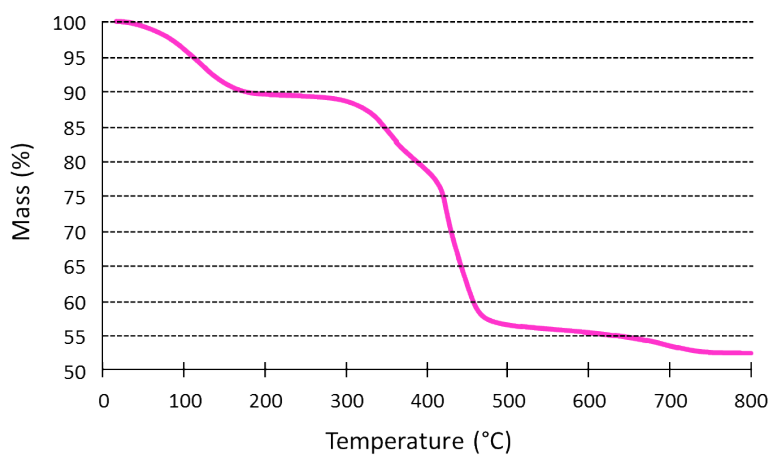

Compound $6\left[\mathrm{Lu}_{2}\left(\mathrm{C}_{2} \mathrm{O}_{4}\right)_{3}\left(\mathrm{H}_{2} \mathrm{O}\right)_{4}\right] \cdot 2 \mathrm{H}_{2} \mathrm{O}$ 
Figure S3. HT-DRX patterns in air of 1-La, 2-Pr, 2-Nd, 3-Eu, 3-Gd, 3-Tb, 3-Dy, 3-Ho, 4-Er, 4-Tm, 5-Yb and 6-Lu lanthanide oxalates lanthanide oxalates.

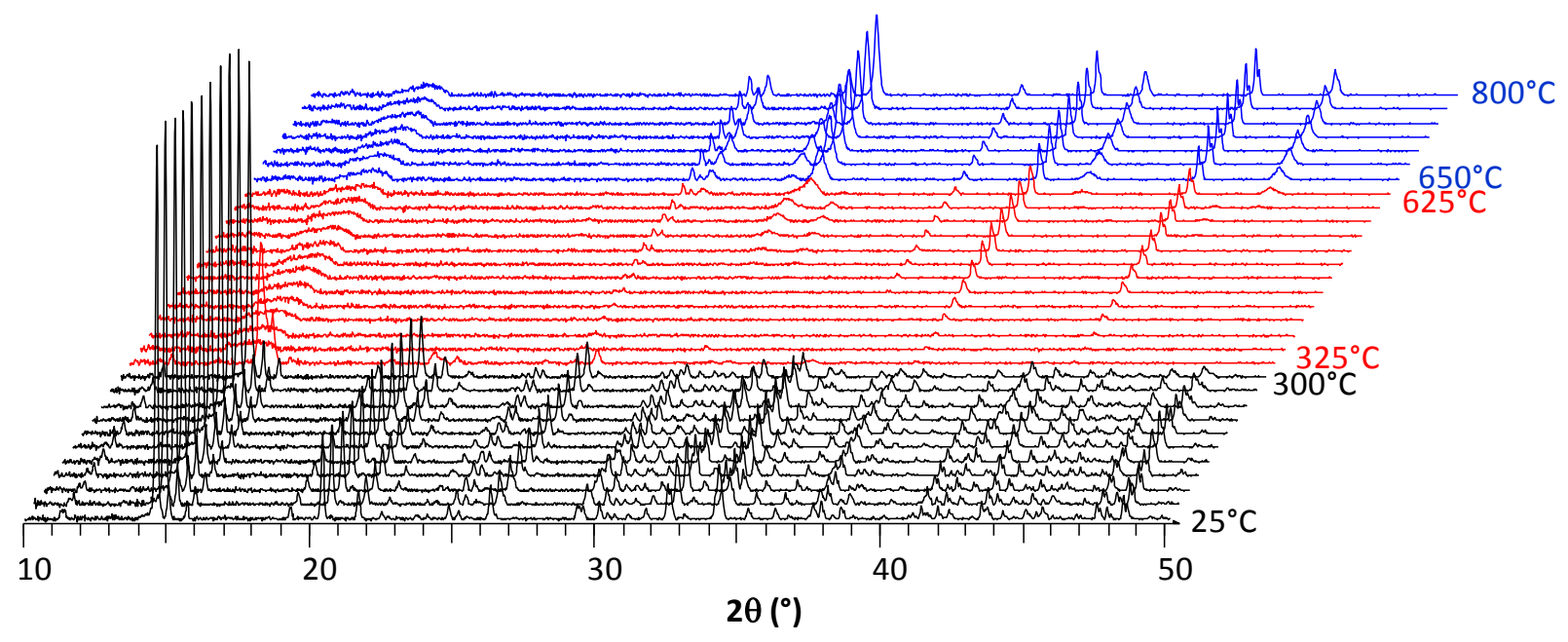

Compound $1\left(\mathrm{~N}_{2} \mathrm{H}_{5}\right) \mathrm{La}\left(\mathrm{C}_{2} \mathrm{O}_{4}\right)_{2}$

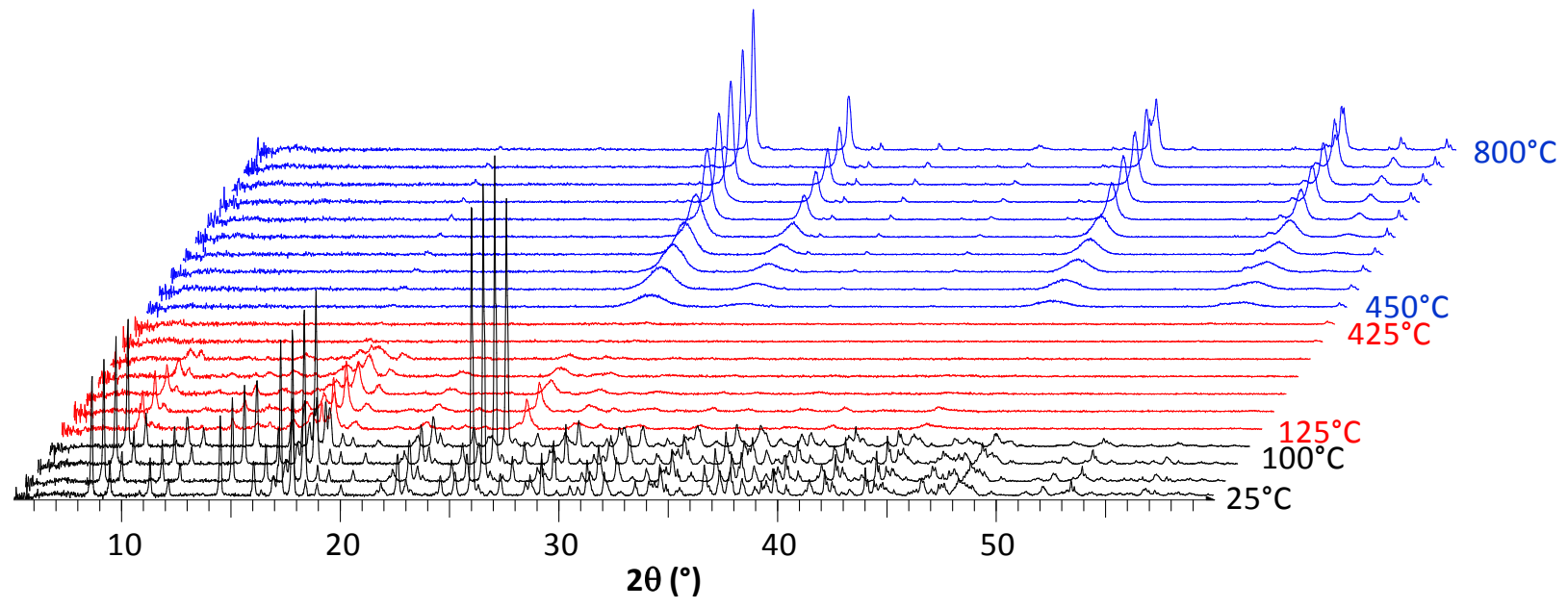

Compound 2-Pr $\mathrm{N}_{2} \mathrm{H}_{5}\left[\left\{\mathrm{Pr}_{2}\left(\mathrm{~N}_{2} \mathrm{H}_{5}\right)\right\}\left(\mathrm{C}_{2} \mathrm{O}_{4}\right)_{4}\right] \cdot 4 \mathrm{H} 2 \mathrm{O}$

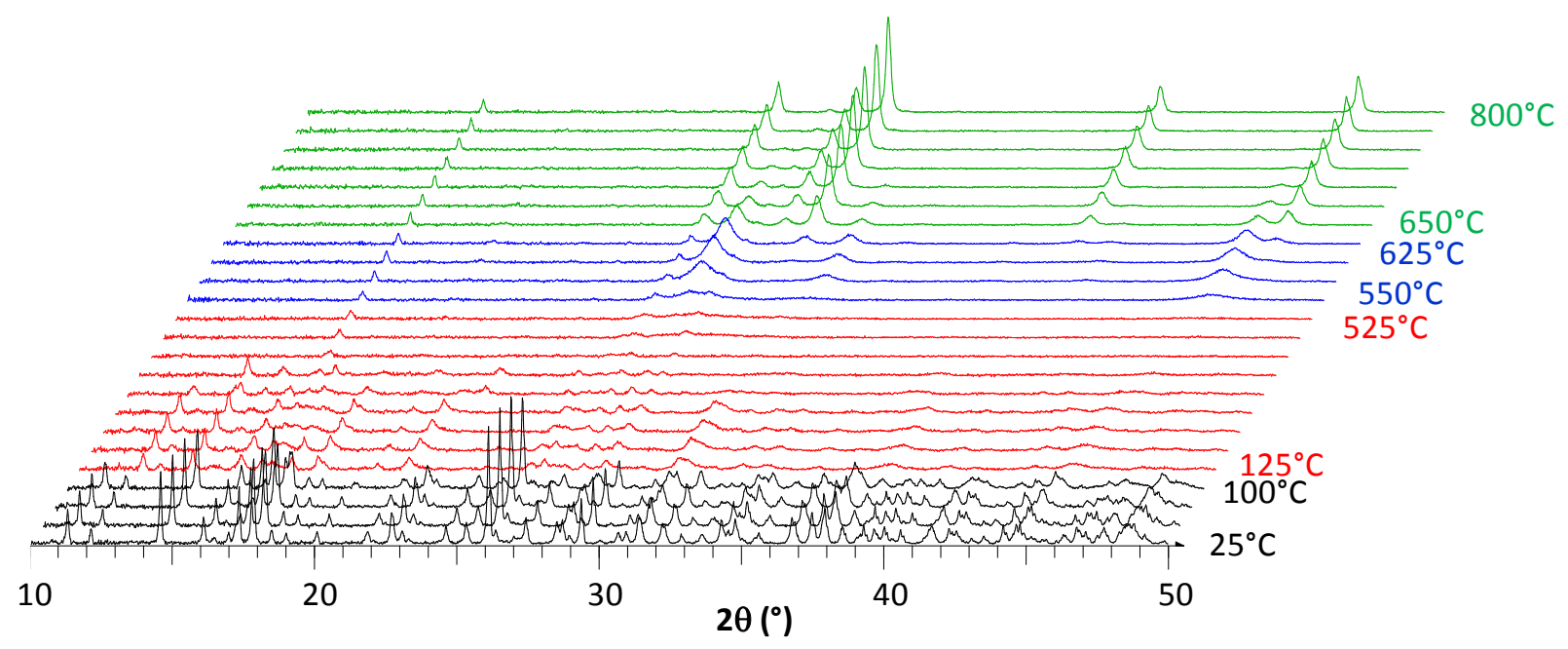


Compound 2-Nd $\mathrm{N}_{2} \mathrm{H}_{5}\left[\left\{\mathrm{Nd}_{2}\left(\mathrm{~N}_{2} \mathrm{H}_{5}\right)\right\}\left(\mathrm{C}_{2} \mathrm{O}_{4}\right)_{4}\right] \cdot 4 \mathrm{H}_{2} \mathrm{O}$

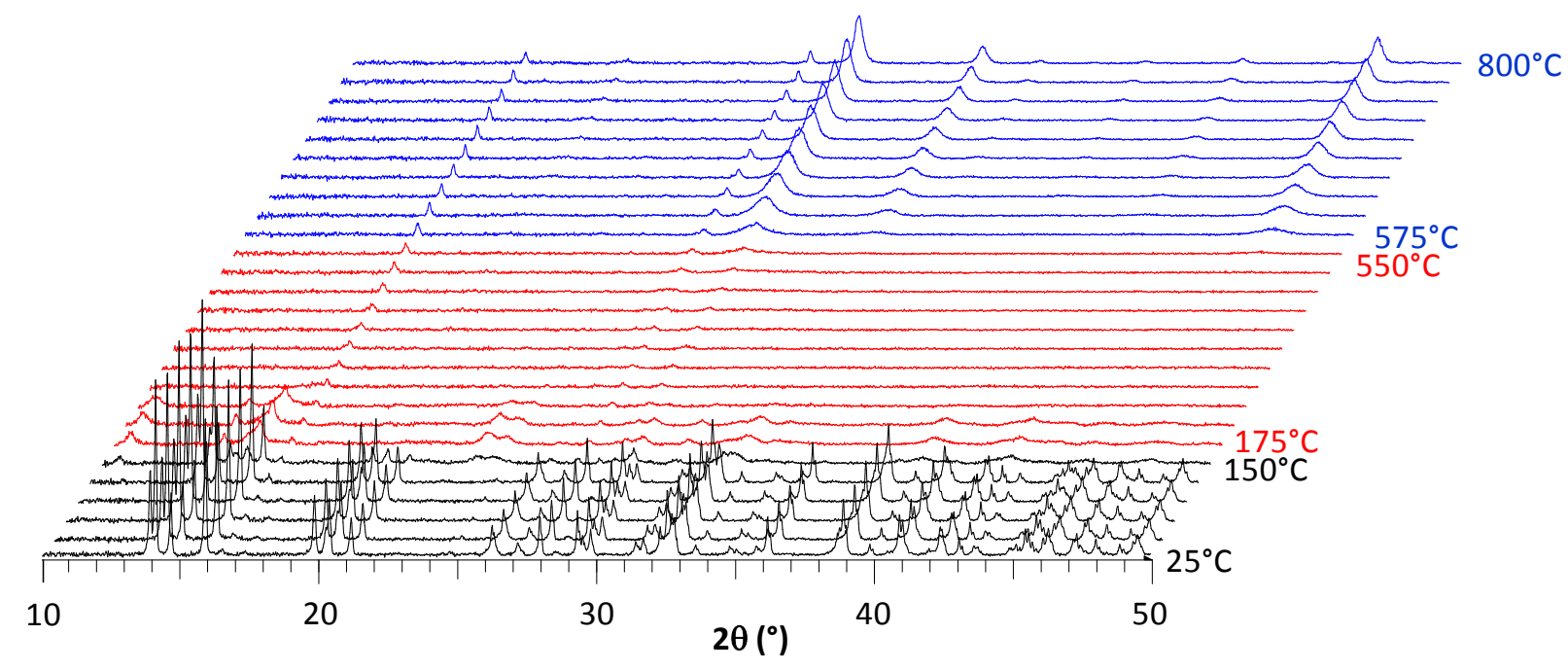

Compound 3-Eu $\left(\mathrm{N}_{2} \mathrm{H}_{5}\right) \mathrm{Eu}\left(\mathrm{C}_{2} \mathrm{O}_{4}\right)_{2} \cdot \mathrm{H}_{2} \mathrm{O}$

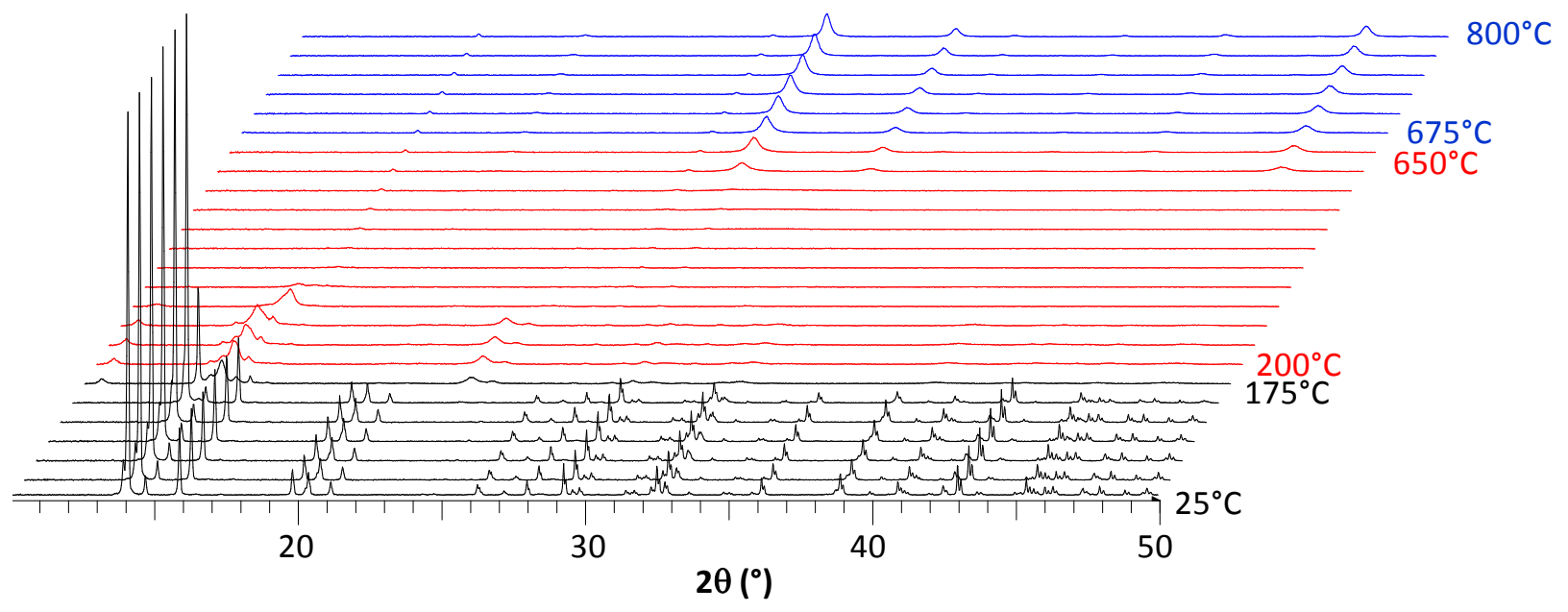

Compound 3-Gd $\left(\mathrm{N}_{2} \mathrm{H}_{5}\right) \mathrm{Gd}\left(\mathrm{C}_{2} \mathrm{O}_{4}\right)_{2} \cdot \mathrm{H}_{2} \mathrm{O}$ 


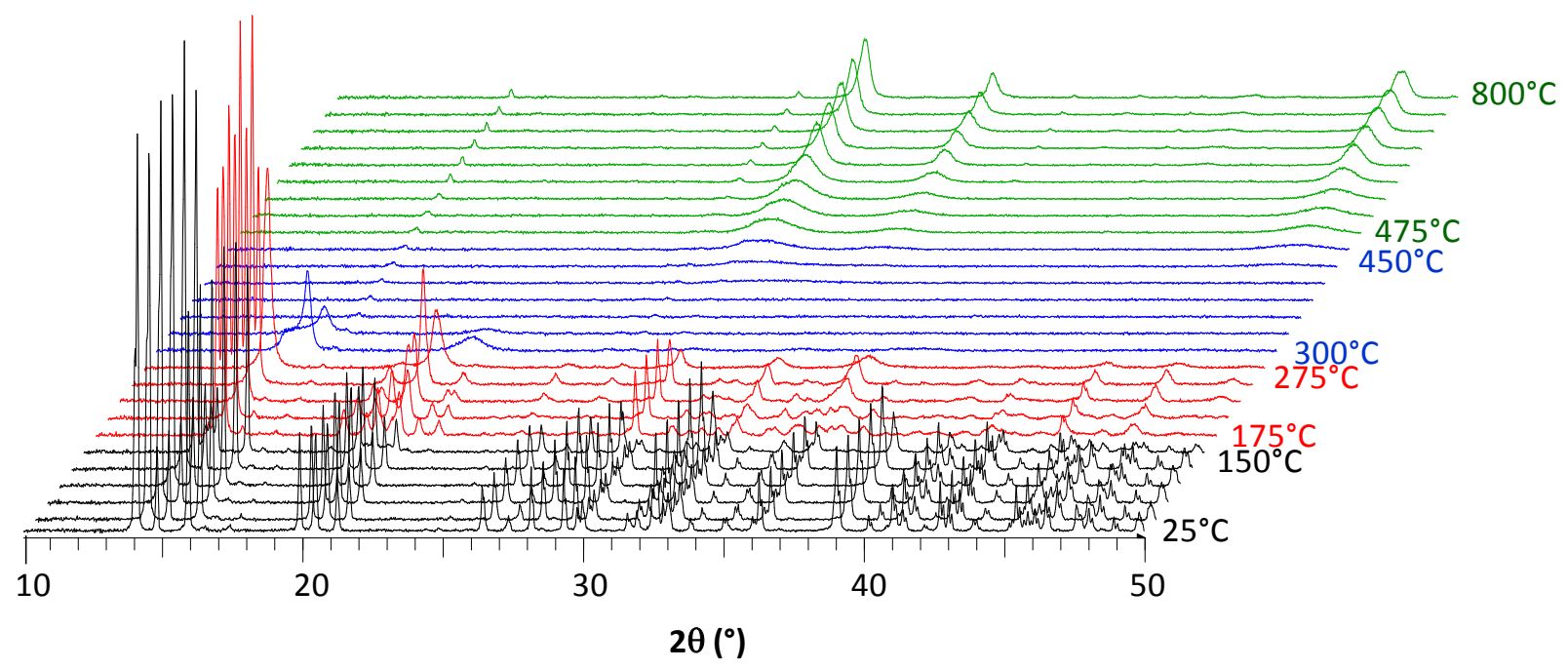

Compound 3-Tb $\left(\mathrm{N}_{2} \mathrm{H}_{5}\right) \mathrm{Tb}\left(\mathrm{C}_{2} \mathrm{O}_{4}\right)_{2} \cdot \mathrm{H}_{2} \mathrm{O}$

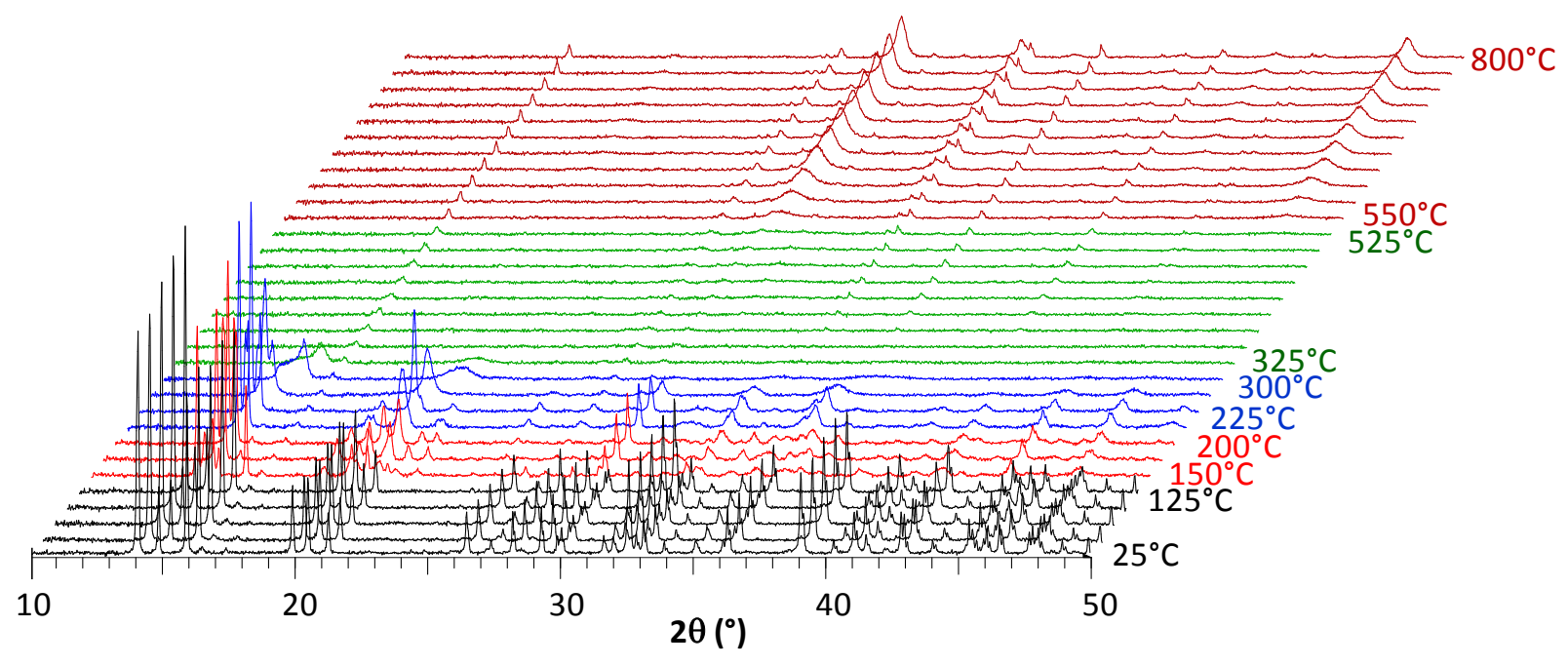

\section{Compound 3-Dy $\left(\mathrm{N}_{2} \mathrm{H}_{5}\right) \mathrm{Dy}\left(\mathrm{C}_{2} \mathrm{O}_{4}\right)_{2} \cdot \mathrm{H}_{2} \mathrm{O}$}

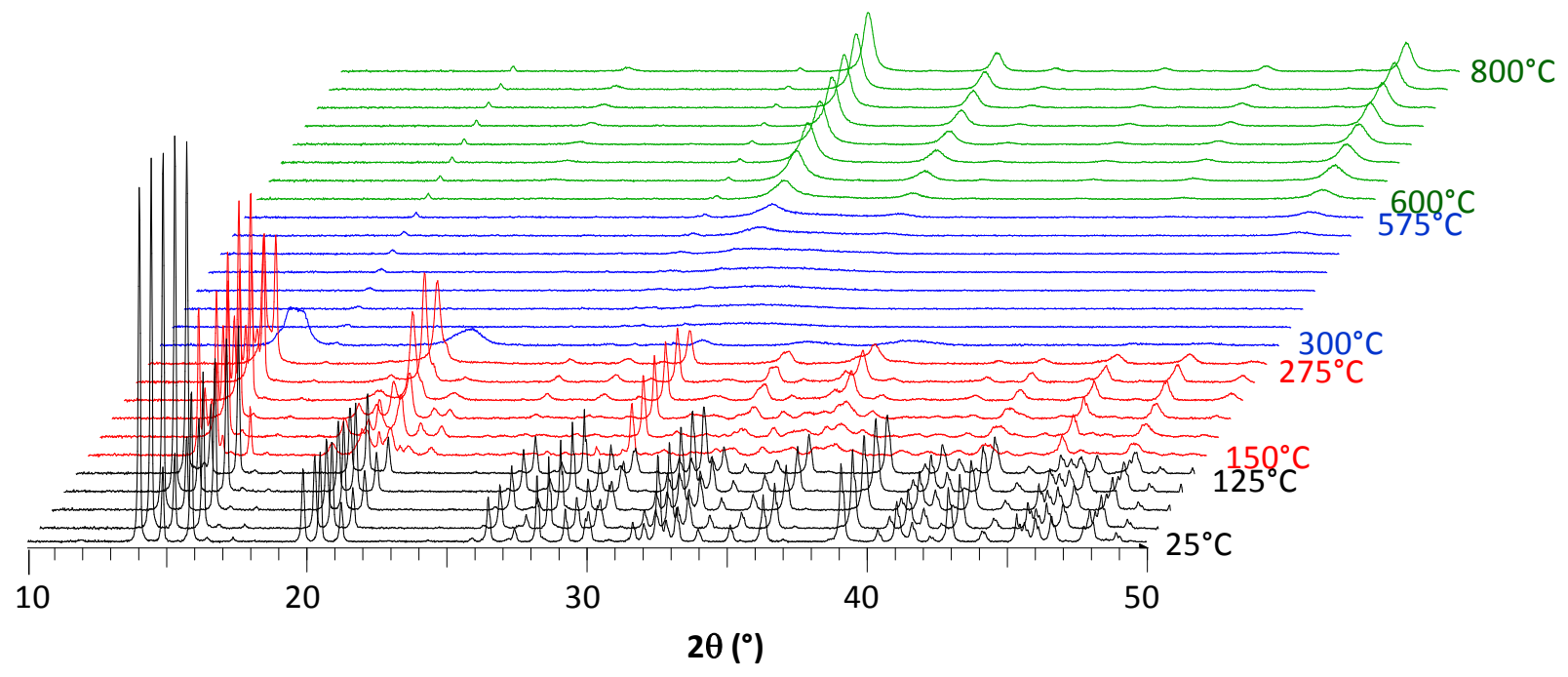

Compound 3-Ho $\left(\mathrm{N}_{2} \mathrm{H}_{5}\right) \mathrm{Ho}\left(\mathrm{C}_{2} \mathrm{O}_{4}\right)_{2} \cdot \mathrm{H}_{2} \mathrm{O}$ 


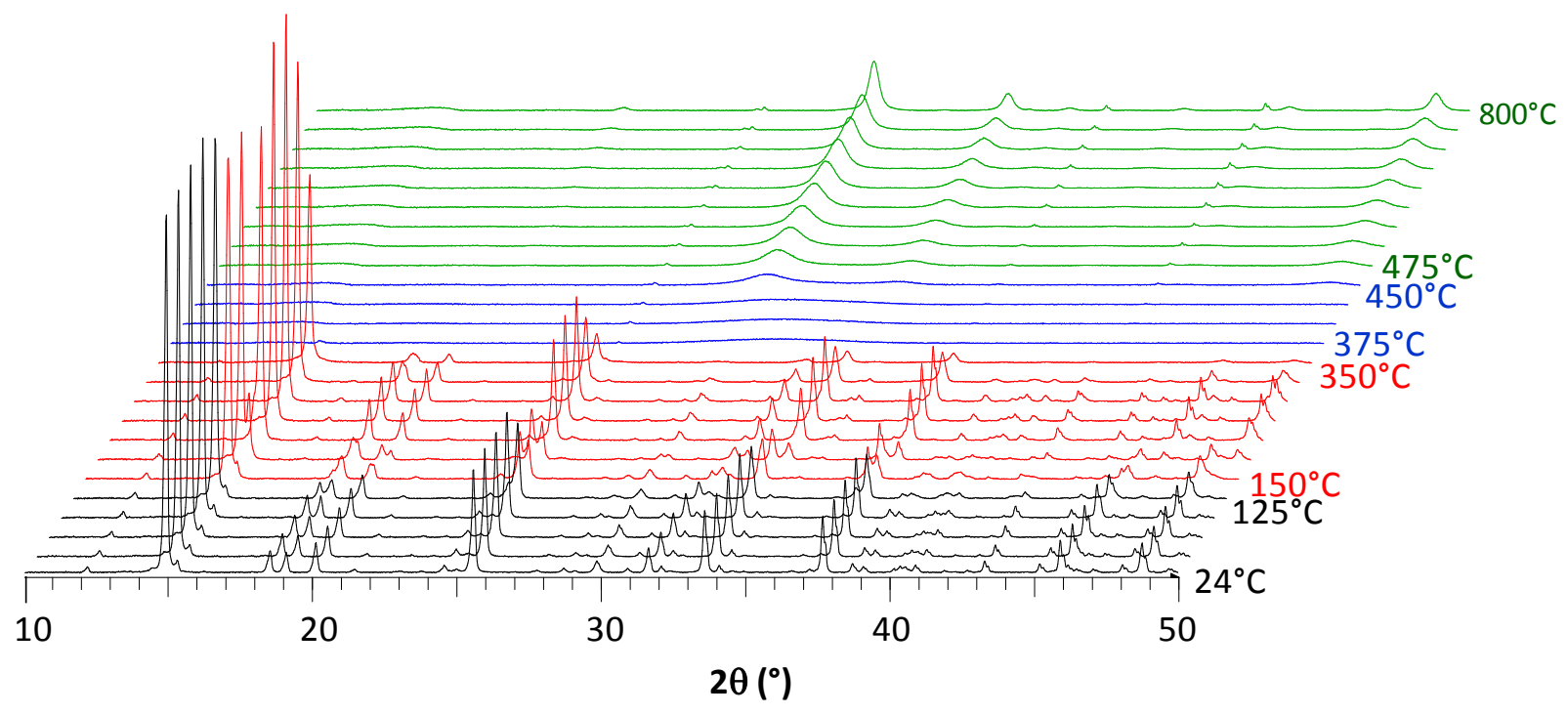

\section{Compound 4-Tm $\left[\left\{\mathrm{Tm}\left(\mathrm{H}_{2} \mathrm{O}\right)\right\}_{2}\left(\mathrm{C}_{2} \mathrm{O}_{4}\right)_{3}\right] \cdot \mathrm{H}_{2} \mathrm{O}$}

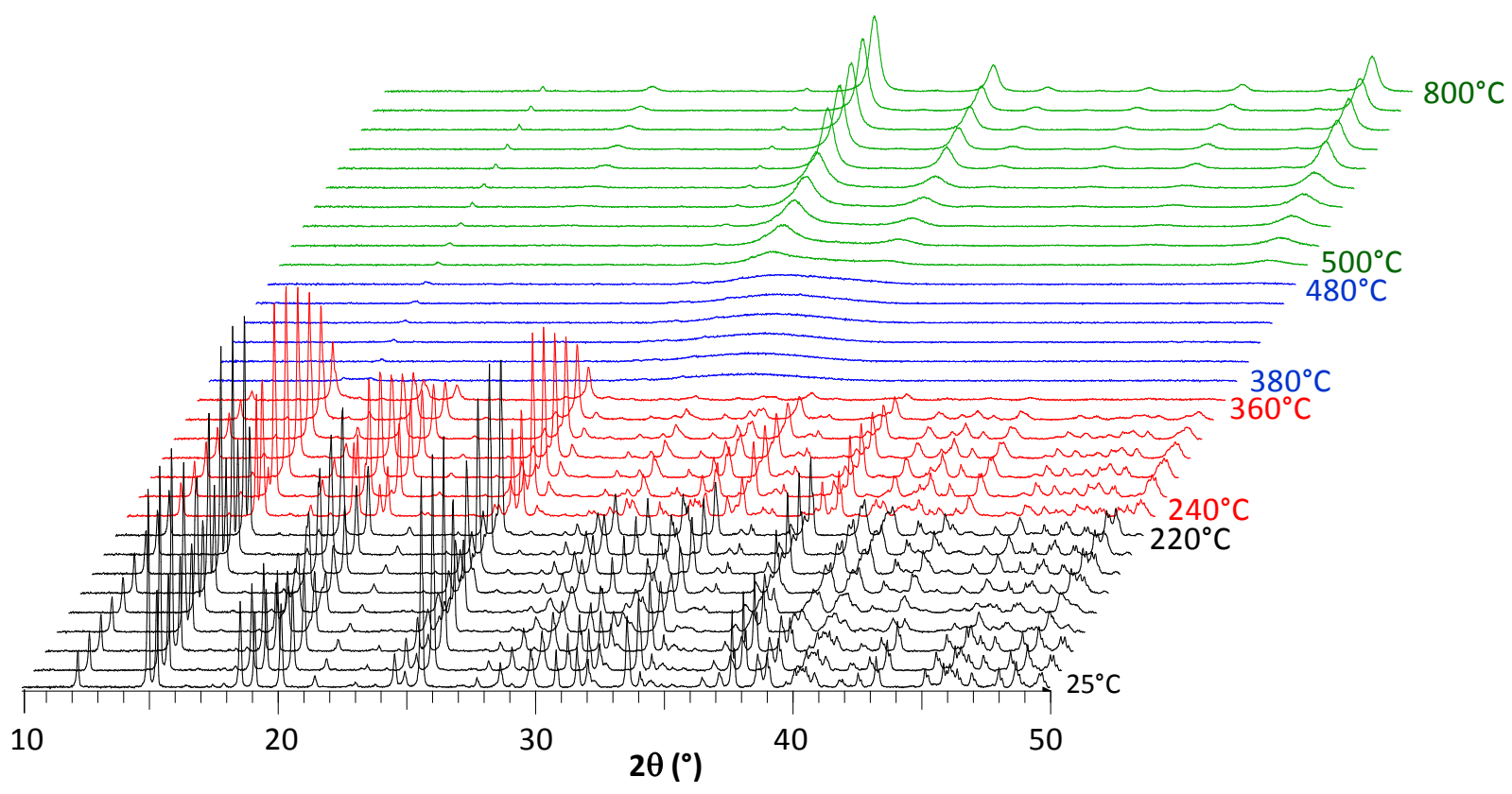

Compound 4-Er $\left[\left\{\operatorname{Er}\left(\mathrm{H}_{2} \mathrm{O}\right)\right\}_{2}\left(\mathrm{C}_{2} \mathrm{O}_{4}\right)_{3}\right] \cdot \mathrm{H}_{2} \mathrm{O}$ 


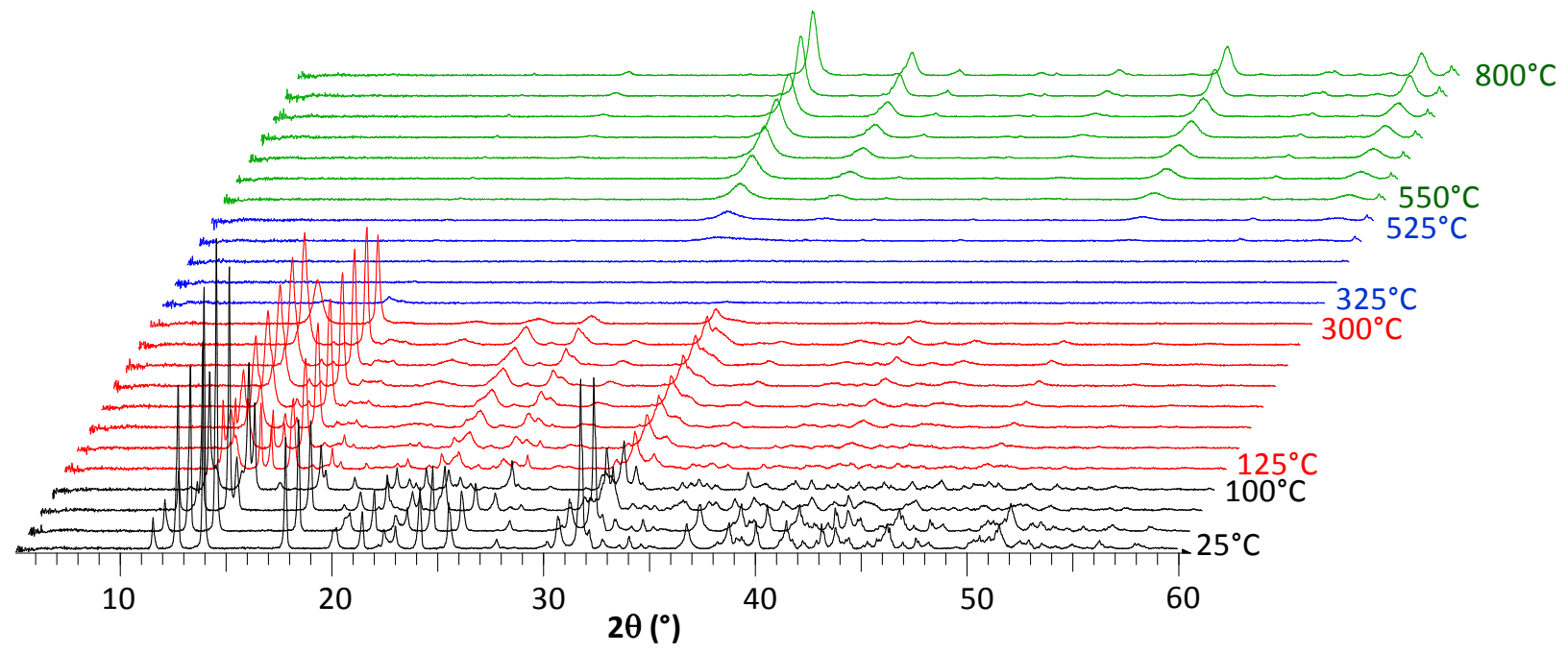

Compound 5- $\mathrm{Yb}\left(\mathrm{N}_{2} \mathrm{H}_{5}\right) \mathrm{Yb}\left(\mathrm{C}_{2} \mathrm{O}_{4}\right)_{2} \cdot 3 \mathrm{H}_{2} \mathrm{O}$

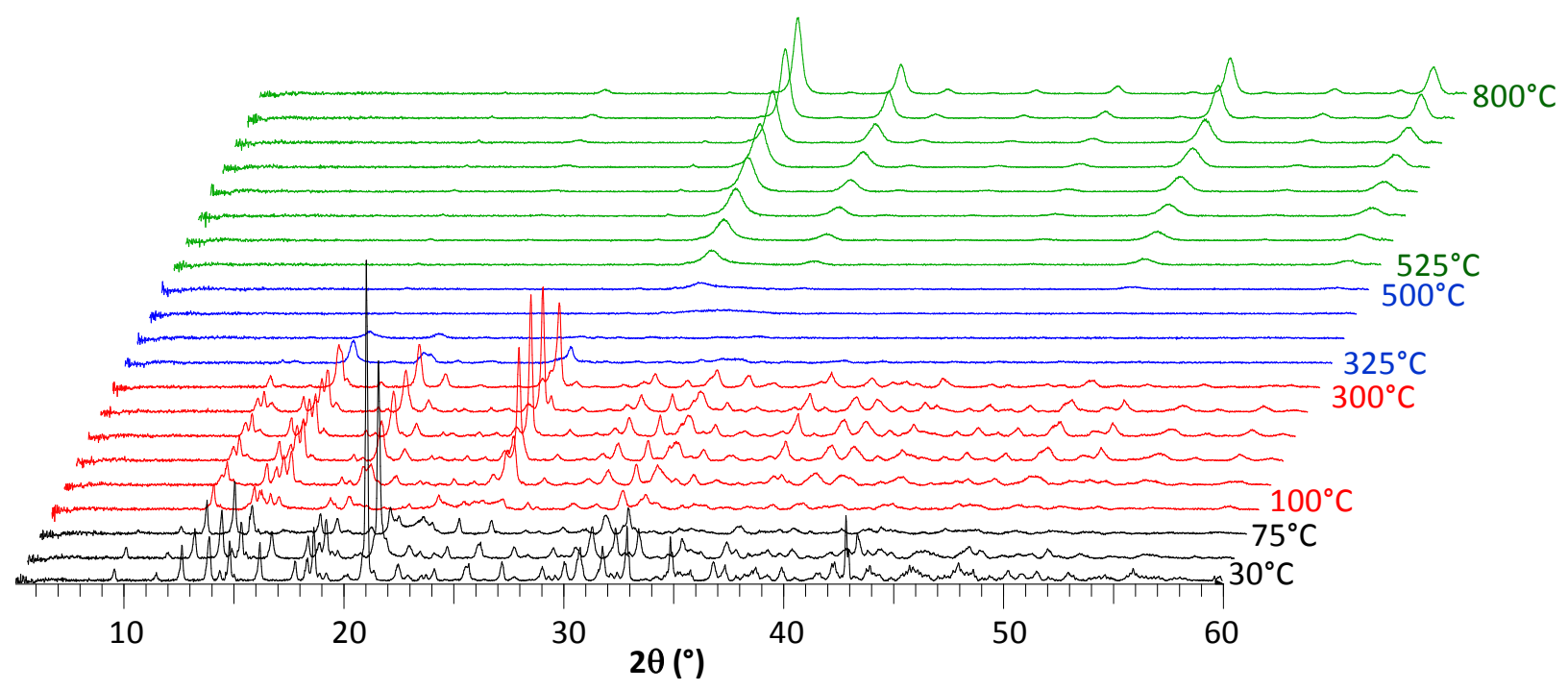

Compound 6- $\mathrm{Lu}\left[\mathrm{Lu}_{2}\left(\mathrm{C}_{2} \mathrm{O}_{4}\right)_{3}\left(\mathrm{H}_{2} \mathrm{O}\right)_{4}\right] \cdot 2 \mathrm{H}_{2} \mathrm{O}$ 
Figure S4. IR spectra of 1-La, 2-Ce, 2-Pr, 2-Nd, 3-Sm, 3-Eu, 3-Gd, 3-Tb, 3-Dy, 3Ho, 5-Yb lanthanide oxalates with assignments of principal bands of oxalate and hydrazinium ions, and of 4-Er and 4-Tm, 6-Lu lanthanide oxalates with assignments of principal bands of oxalate ion.

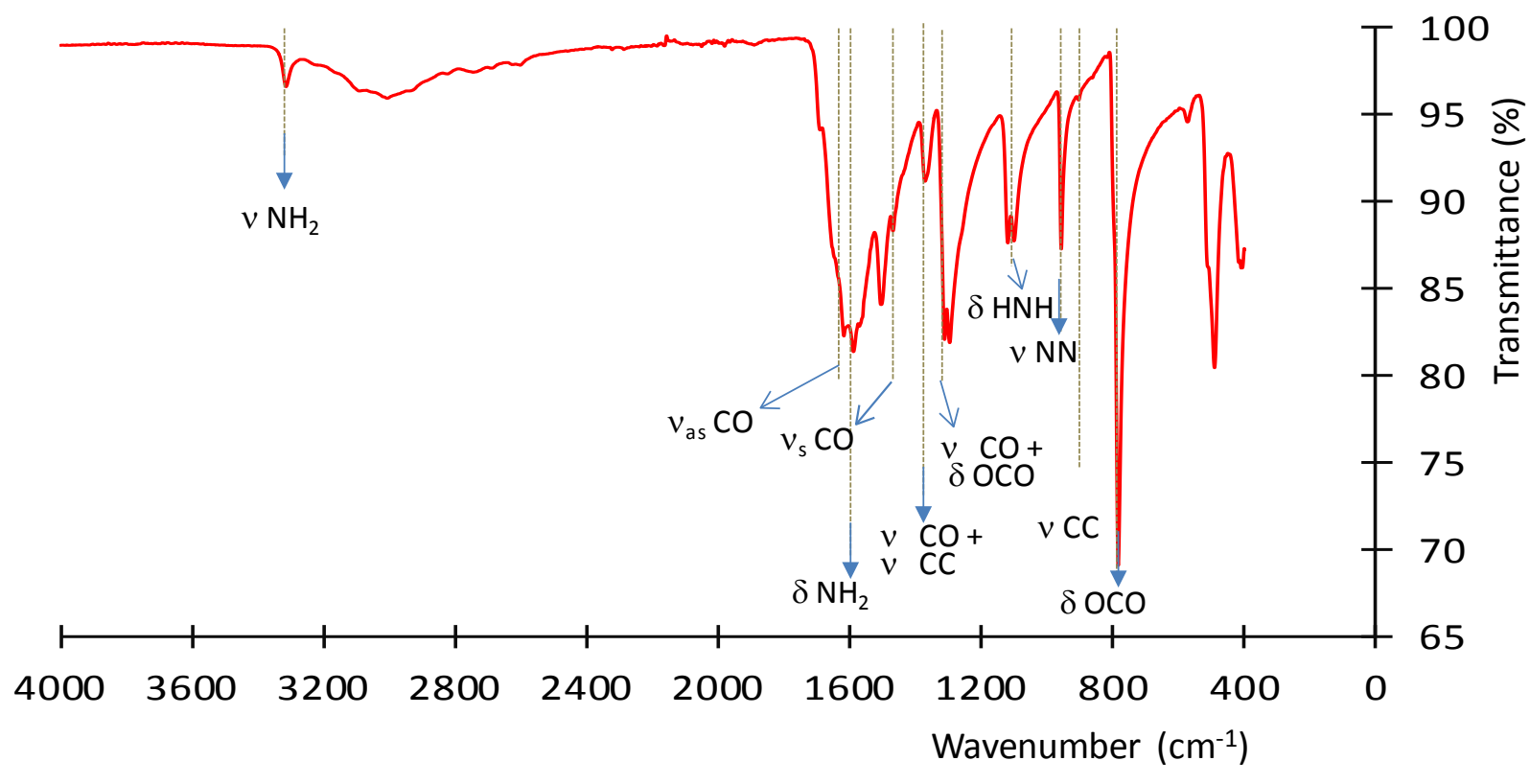

Compound 1-La (1) $\mathrm{N}_{2} \mathrm{H}_{5}\left[\mathrm{La}\left(\mathrm{C}_{2} \mathrm{O}_{4}\right)_{2}\right]$

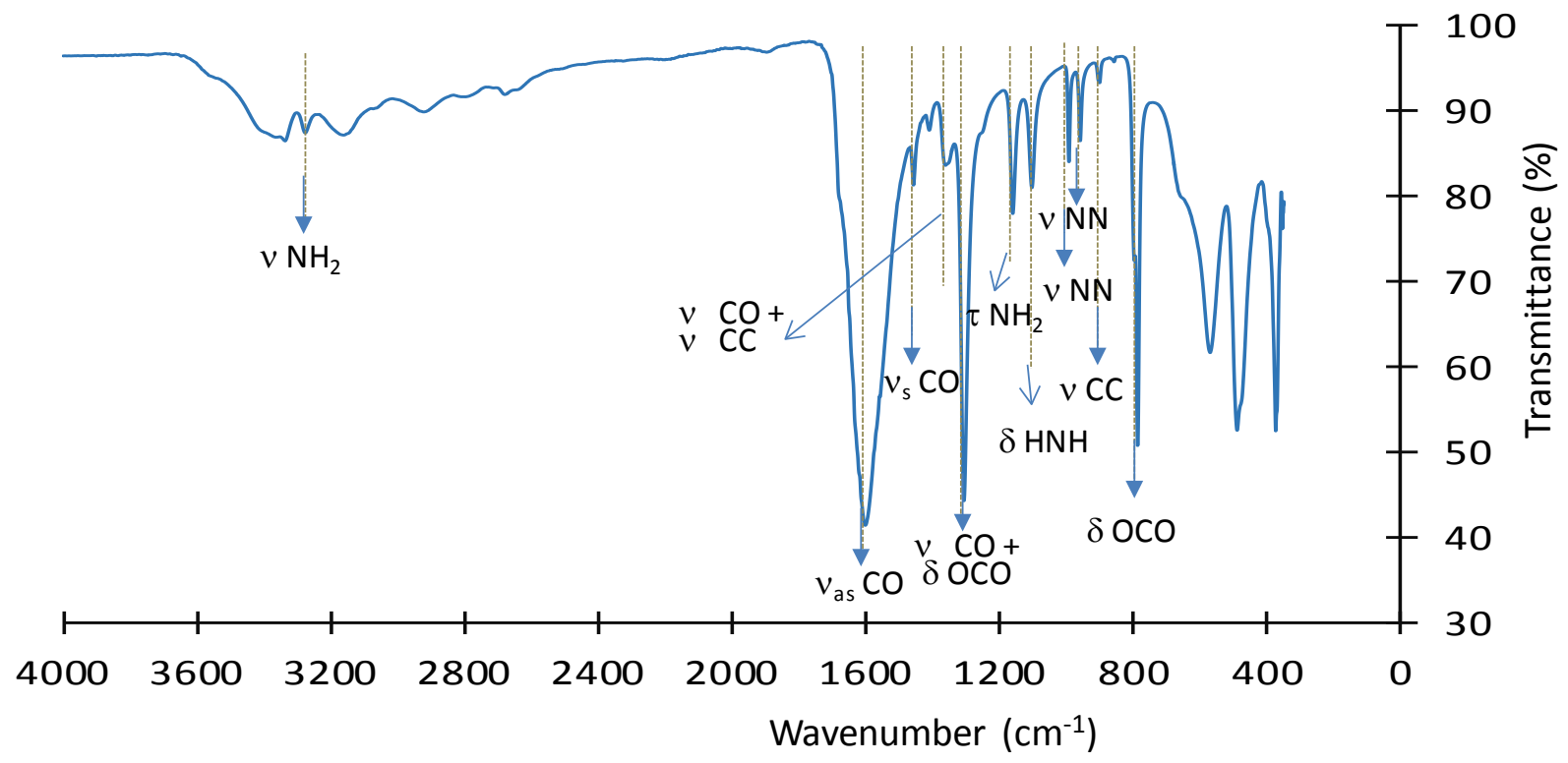

Compound 2-Ce $\mathrm{N}_{2} \mathrm{H}_{5}\left[\left\{\mathrm{Ce}_{2}\left(\mathrm{~N}_{2} \mathrm{H}_{5}\right)\right\}\left(\mathrm{C}_{2} \mathrm{O}_{4}\right)_{4}\right] \cdot 4 \mathrm{H}_{2} \mathrm{O}$ 


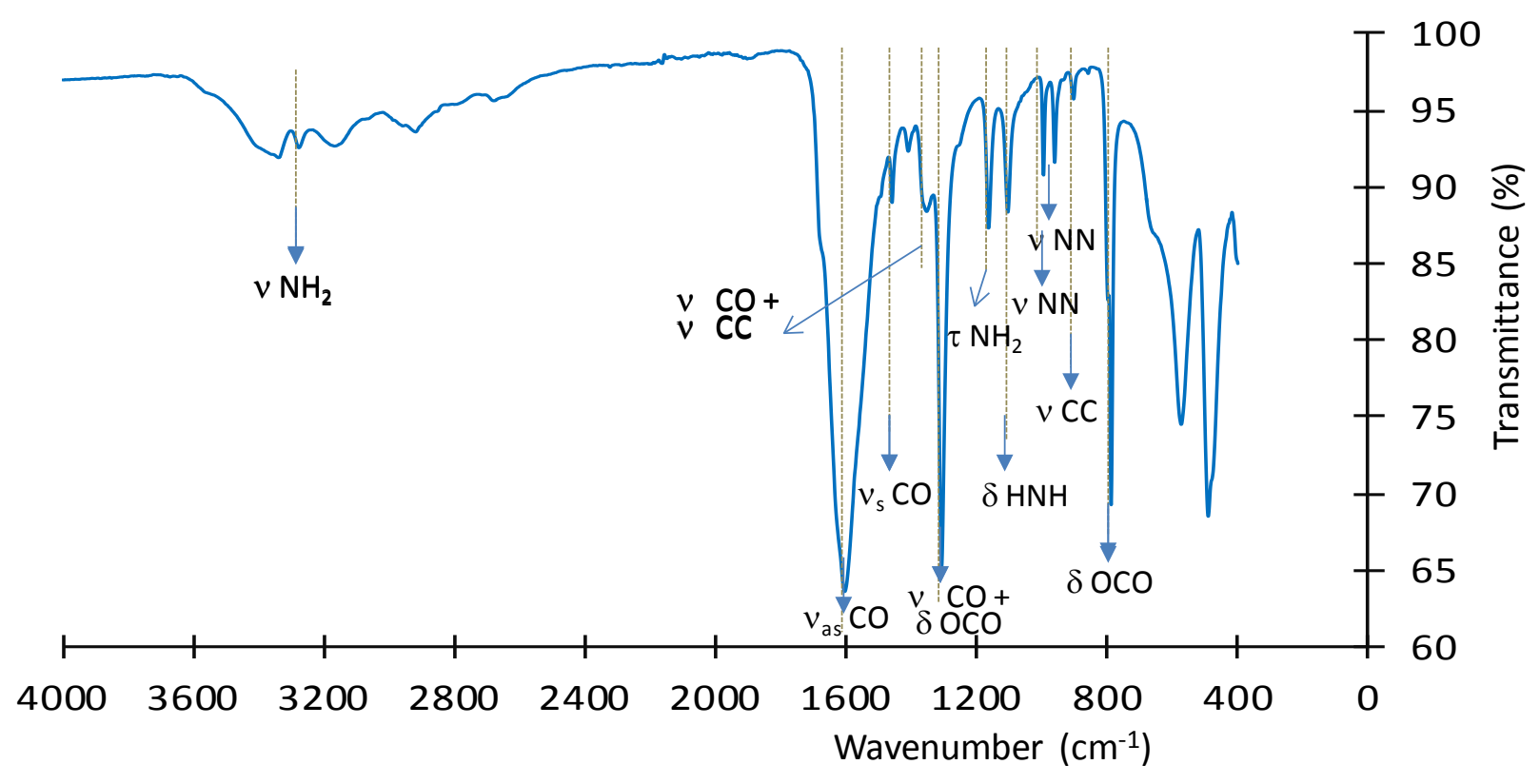

Compound 2-Pr $\mathrm{N}_{2} \mathrm{H}_{5}\left[\left\{\mathrm{Pr}_{2}\left(\mathrm{~N}_{2} \mathrm{H}_{5}\right)\right\}\left(\mathrm{C}_{2} \mathrm{O}_{4}\right)_{4}\right] \cdot 4 \mathrm{H}_{2} \mathrm{O}$

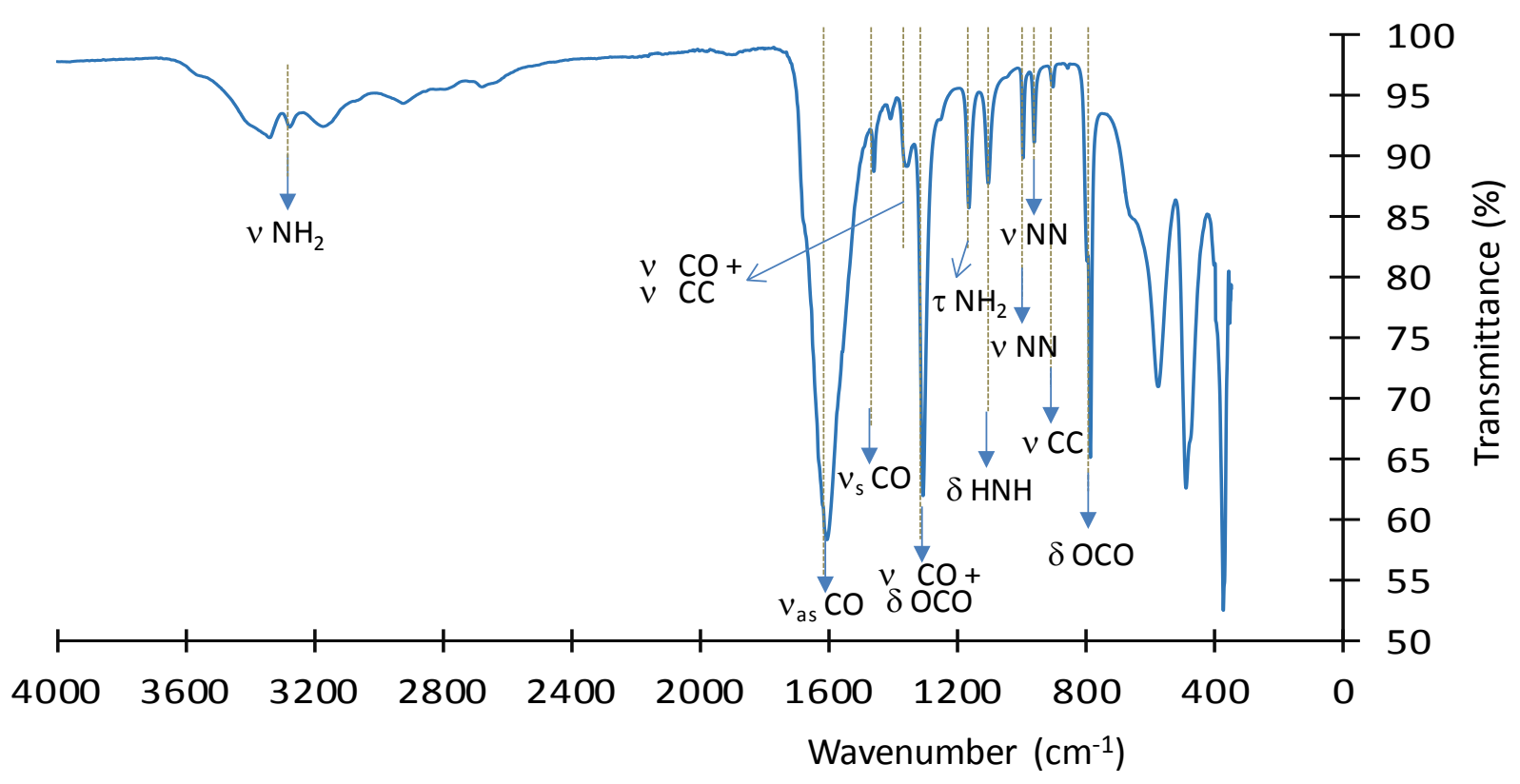

Compound 2-Nd $\mathrm{N}_{2} \mathrm{H}_{5}\left[\left\{\mathrm{Nd}_{2}\left(\mathrm{~N}_{2} \mathrm{H}_{5}\right)\right\}\left(\mathrm{C}_{2} \mathrm{O}_{4}\right)_{4}\right] \cdot 4 \mathrm{H}_{2} \mathrm{O}$ 


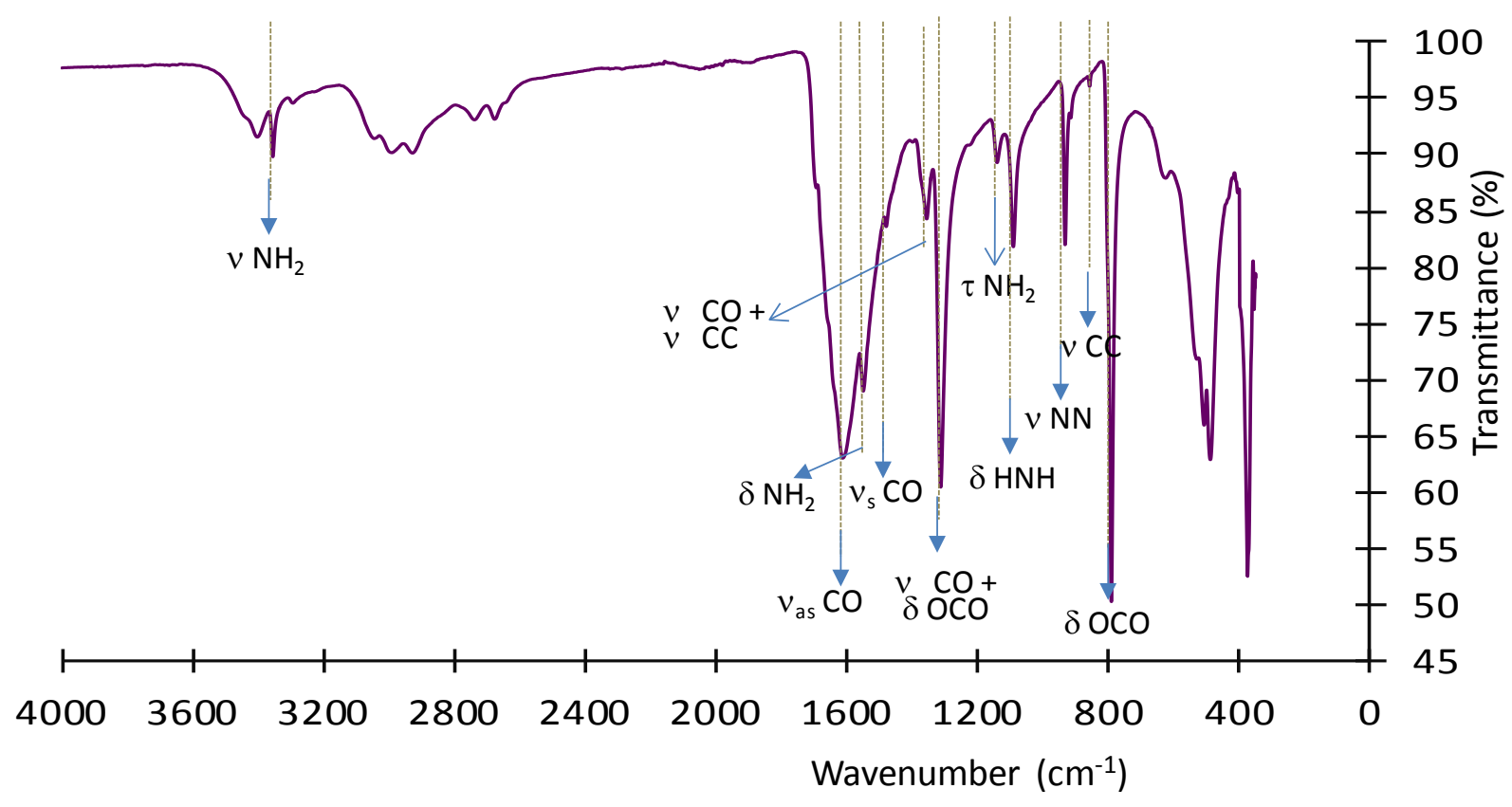

Compound 3-Sm $\left(\mathrm{N}_{2} \mathrm{H}_{5}\right) \mathrm{Sm}\left(\mathrm{C}_{2} \mathrm{O}_{4}\right)_{2} \cdot \mathrm{H}_{2} \mathrm{O}$

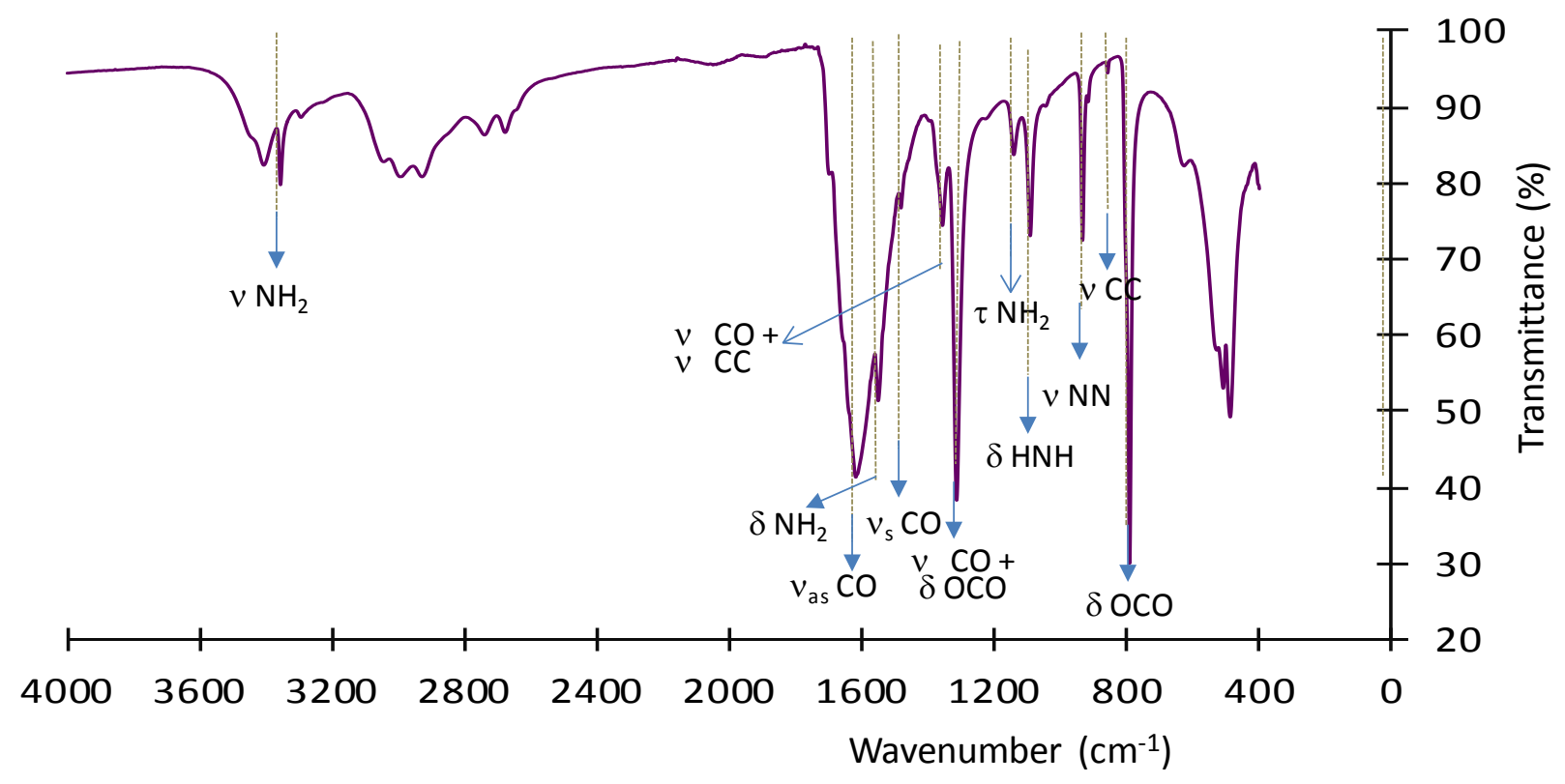

Compound 3-Eu $\left(\mathrm{N}_{2} \mathrm{H}_{5}\right) \mathrm{Eu}\left(\mathrm{C}_{2} \mathrm{O}_{4}\right)_{2} \cdot \mathrm{H}_{2} \mathrm{O}$ 


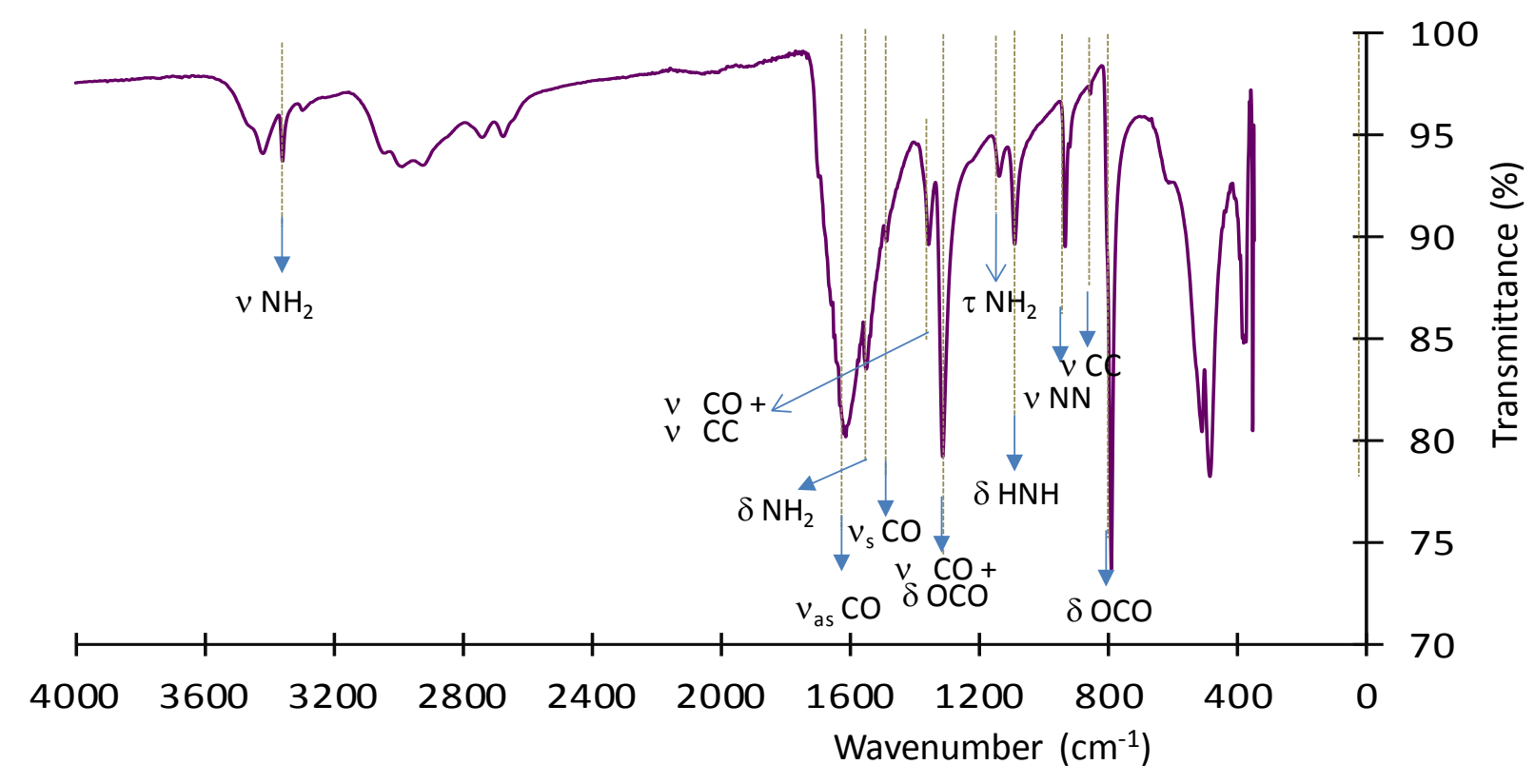

Compound 3-Gd $\left(\mathrm{N}_{2} \mathrm{H}_{5}\right) \mathrm{Gd}\left(\mathrm{C}_{2} \mathrm{O}_{4}\right)_{2} \cdot \mathrm{H}_{2} \mathrm{O}$

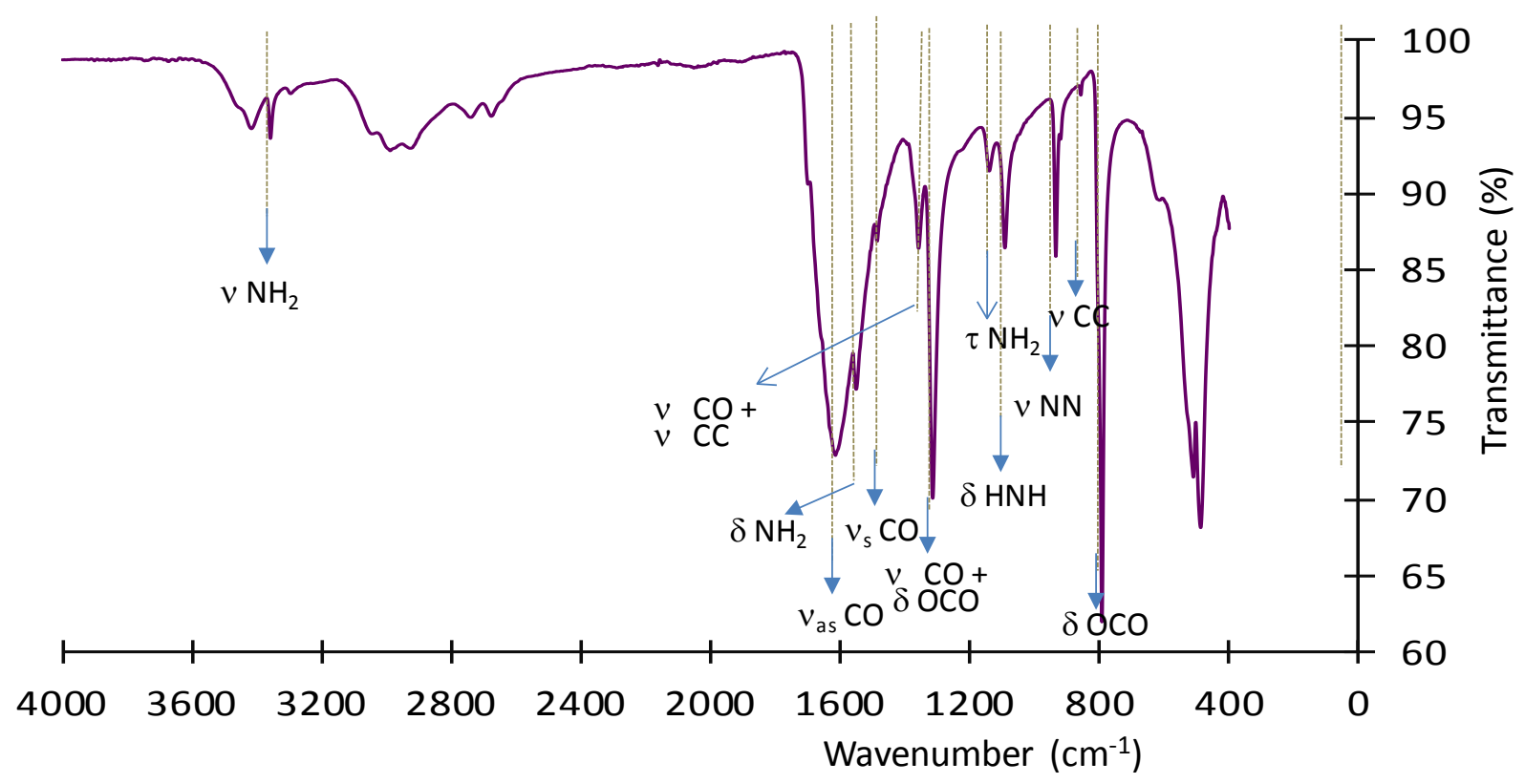

Compound 3-Tb $\left(\mathrm{N}_{2} \mathrm{H}_{5}\right) \mathrm{Tb}\left(\mathrm{C}_{2} \mathrm{O}_{4}\right)_{2} \cdot \mathrm{H}_{2} \mathrm{O}$ 


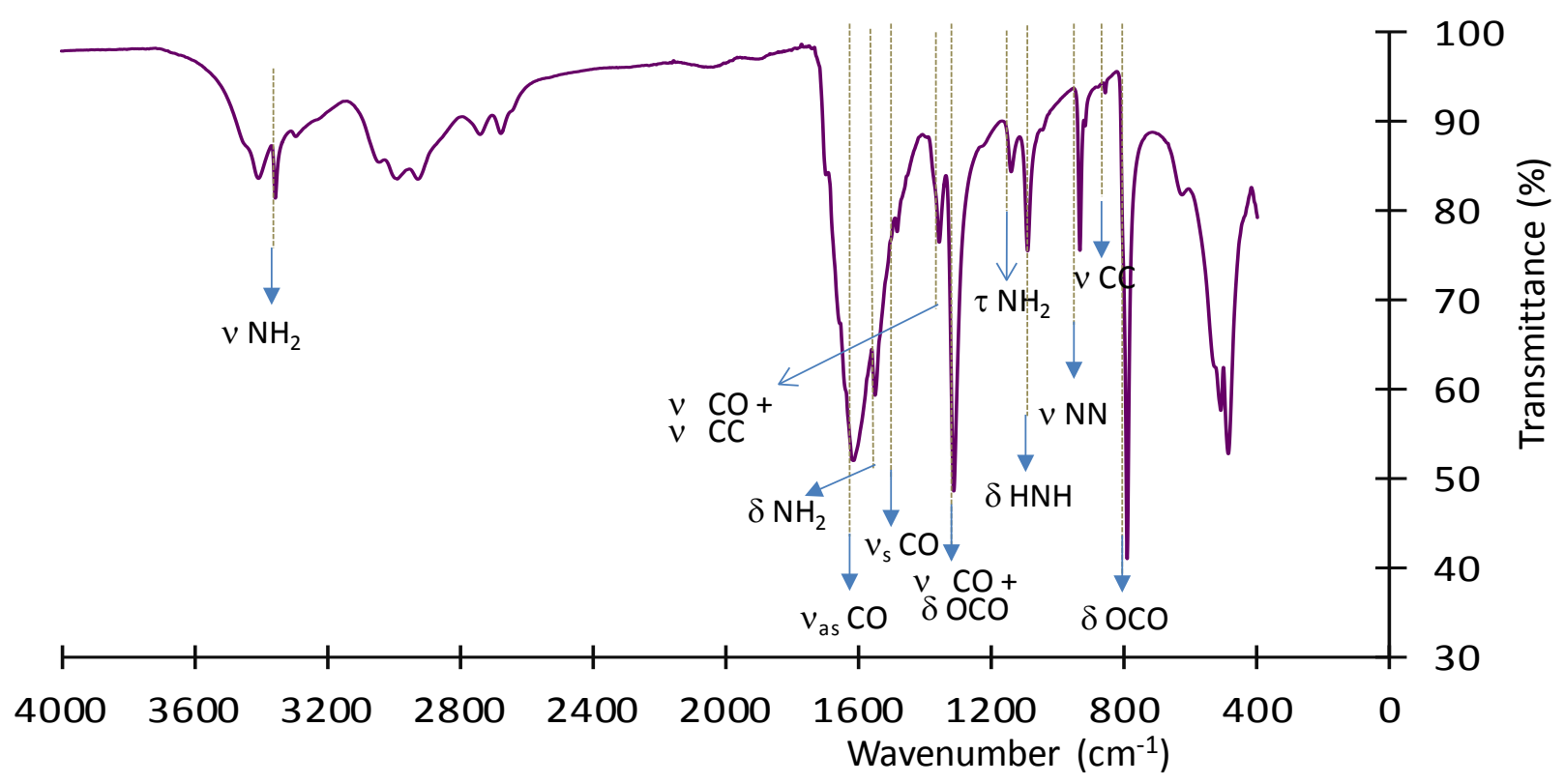

Compound 3-Dy $\left(\mathrm{N}_{2} \mathrm{H}_{5}\right) \mathrm{Dy}\left(\mathrm{C}_{2} \mathrm{O}_{4}\right)_{2} \cdot \mathrm{H}_{2} \mathrm{O}$

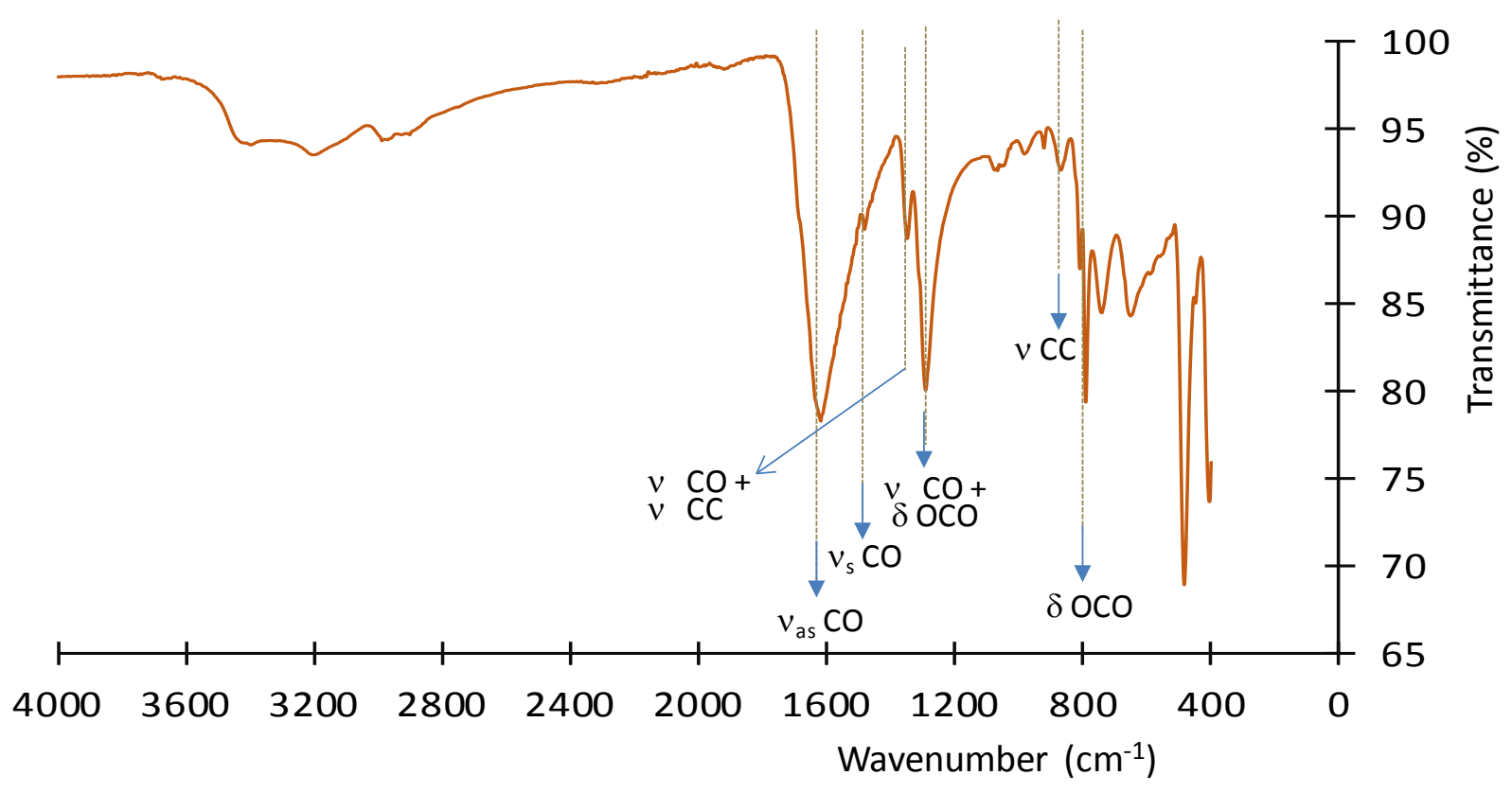

Compound 4-Er $\left[\left\{\mathrm{Er}\left(\mathrm{H}_{2} \mathrm{O}\right)\right\}_{2}\left(\mathrm{C}_{2} \mathrm{O}_{4}\right)_{3}\right] \cdot \mathrm{H}_{2} \mathrm{O}$ 


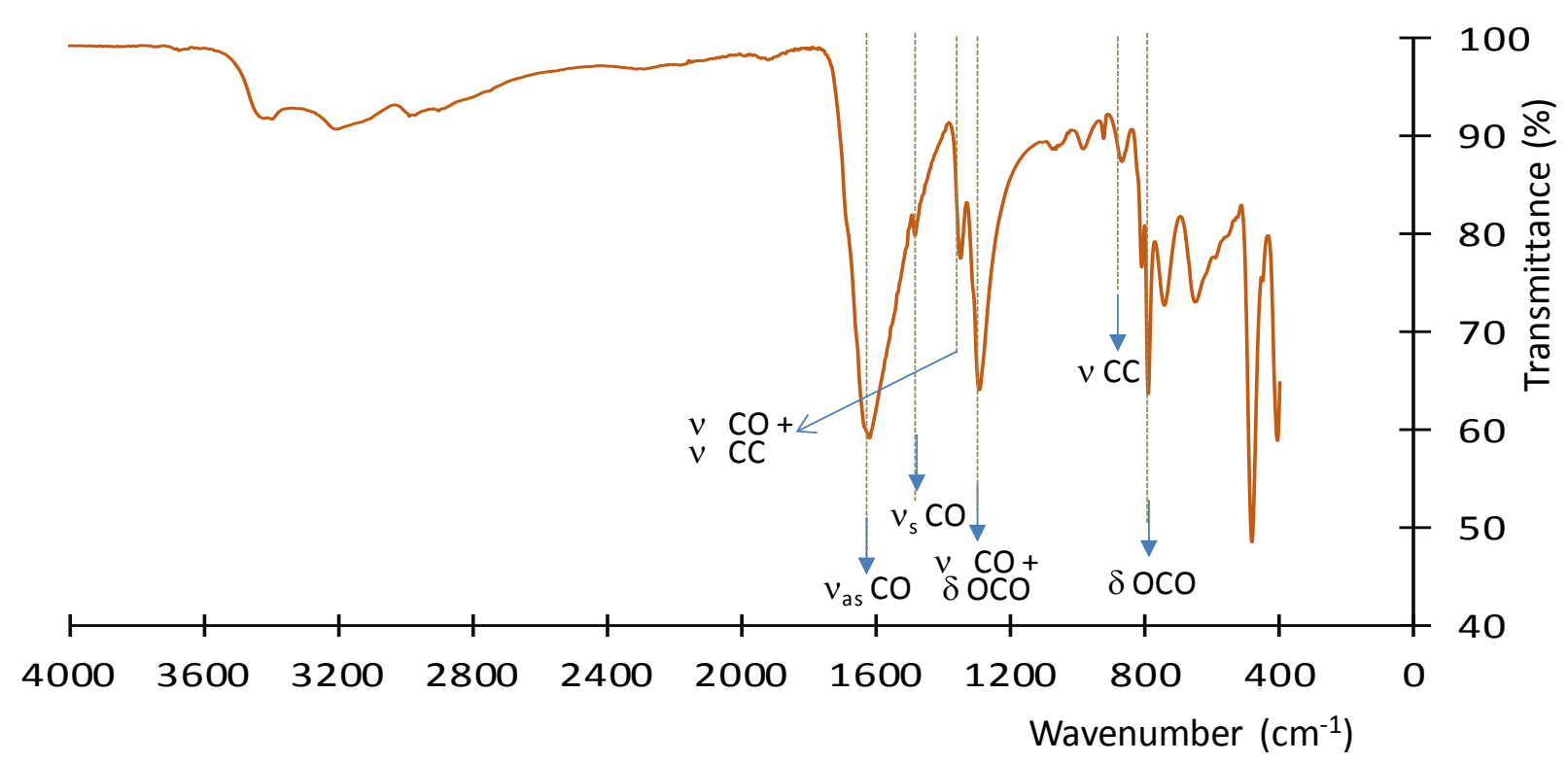

Compound 4-Tm $\left[\left\{\mathrm{Tm}\left(\mathrm{H}_{2} \mathrm{O}\right)\right\}_{2}\left(\mathrm{C}_{2} \mathrm{O}_{4}\right)_{3}\right] \cdot \mathrm{H}_{2} \mathrm{O}$

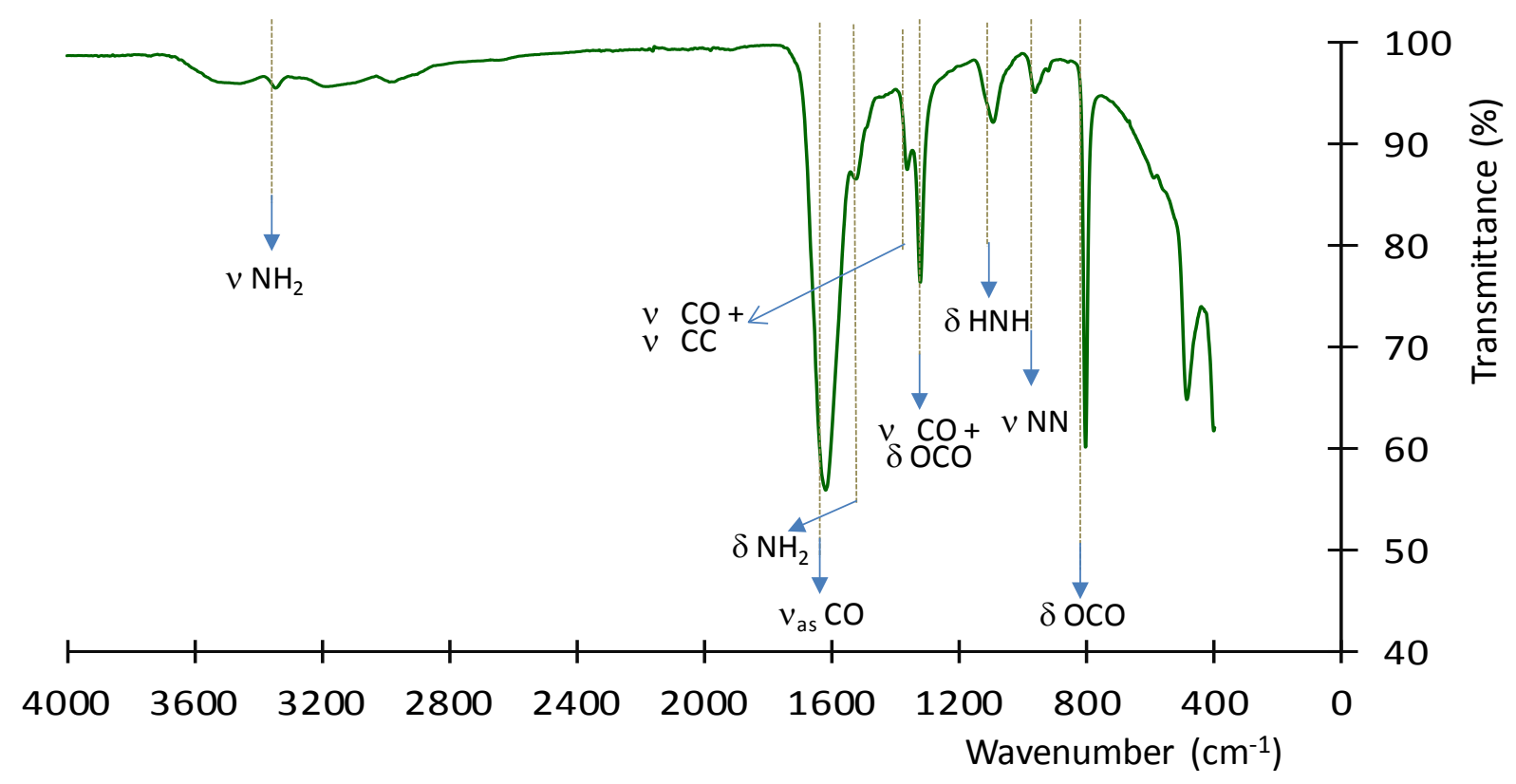

Compound 5-Yb $\left(\mathrm{N}_{2} \mathrm{H}_{5}\right) \mathrm{Yb}\left(\mathrm{C}_{2} \mathrm{O}_{4}\right)_{2} \cdot 3 \mathrm{H}_{2} \mathrm{O}$ 


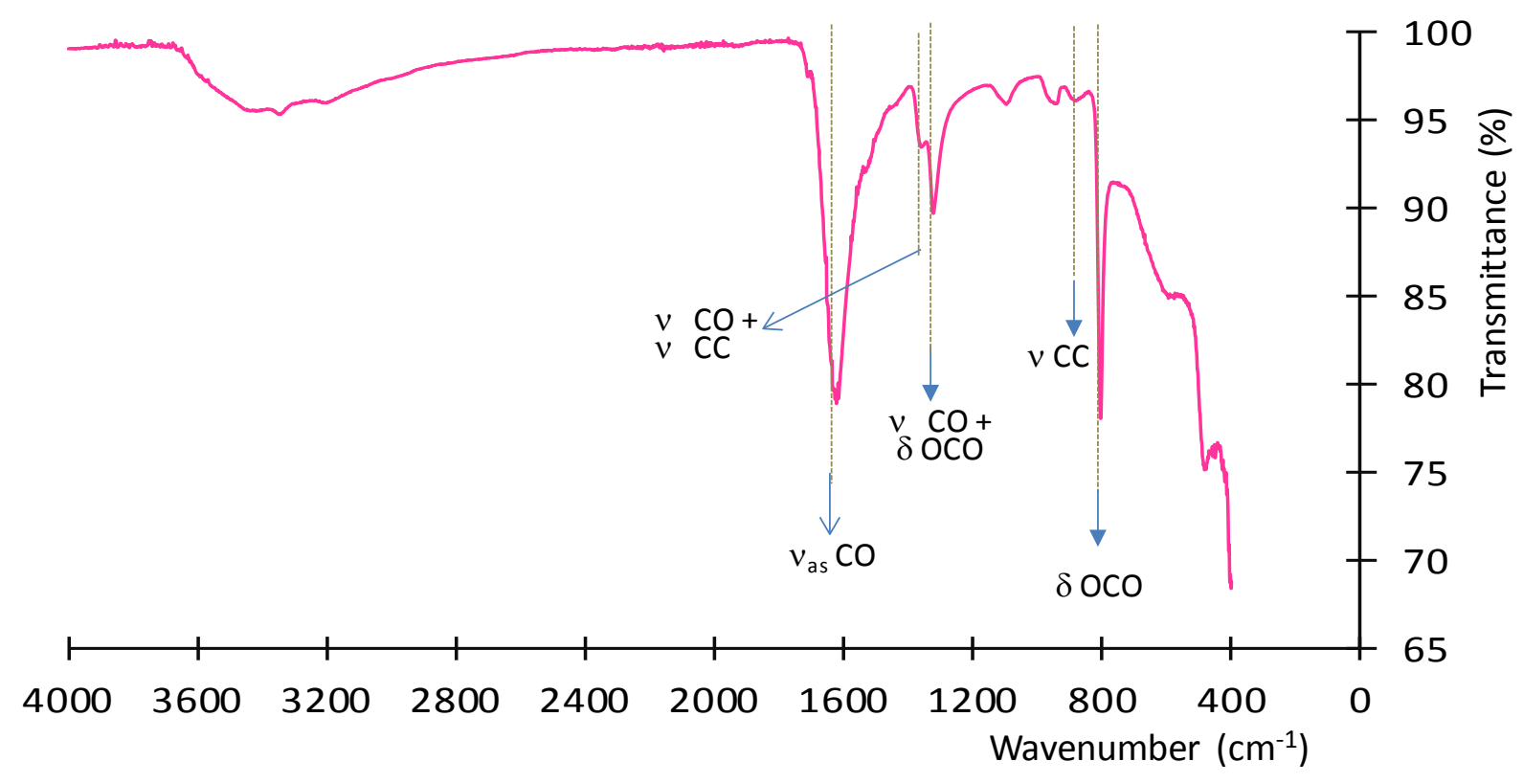

Compound 6- $\mathbf{L u}\left[\mathrm{Lu}_{2}\left(\mathrm{C}_{2} \mathrm{O}_{4}\right)_{3}\left(\mathrm{H}_{2} \mathrm{O}\right)_{4}\right] \cdot 2 \mathrm{H}_{2} \mathrm{O}$

Figure S5. UV-Vis spectra of 2-Pr, 2-Nd, 3-Sm, 3-Eu, 3-Tb, 3-Dy, 3-Ho, 4-Er and 4-Tm lanthanide oxalates.

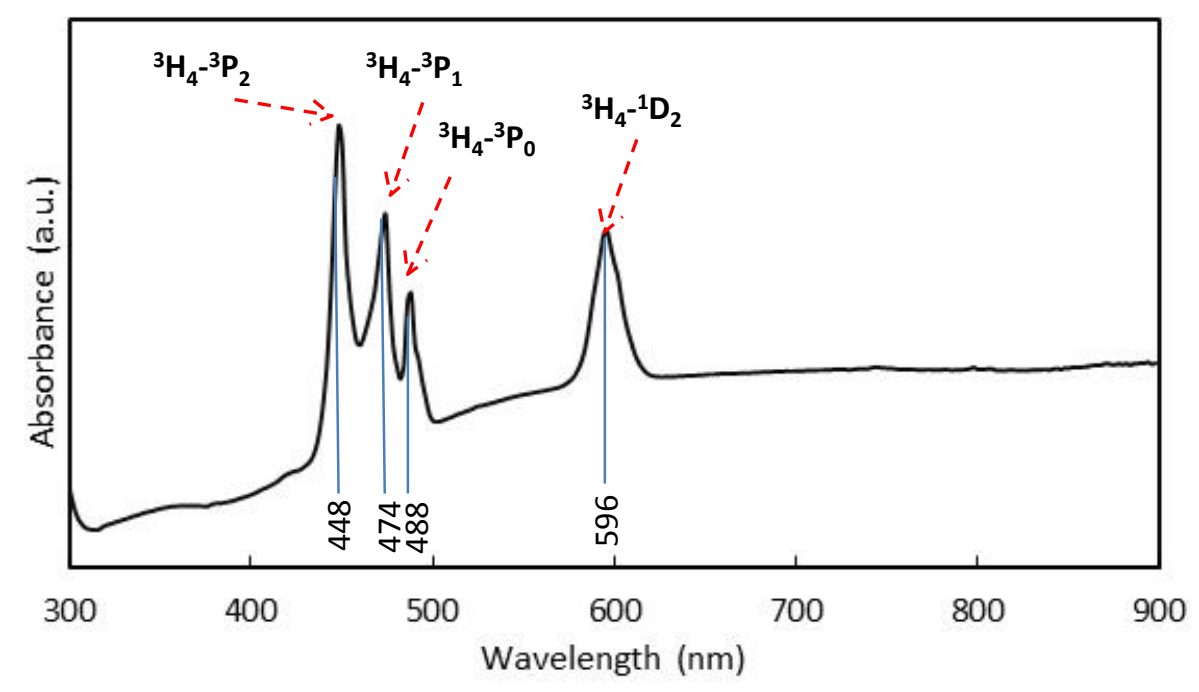

Compound 2-Pr $\mathrm{N}_{2} \mathrm{H}_{5}\left[\left\{\mathrm{Pr}_{2}\left(\mathrm{~N}_{2} \mathrm{H}_{5}\right)\right\}\left(\mathrm{C}_{2} \mathrm{O}_{4}\right)_{4}\right] \cdot 4 \mathrm{H}_{2} \mathrm{O}$ 


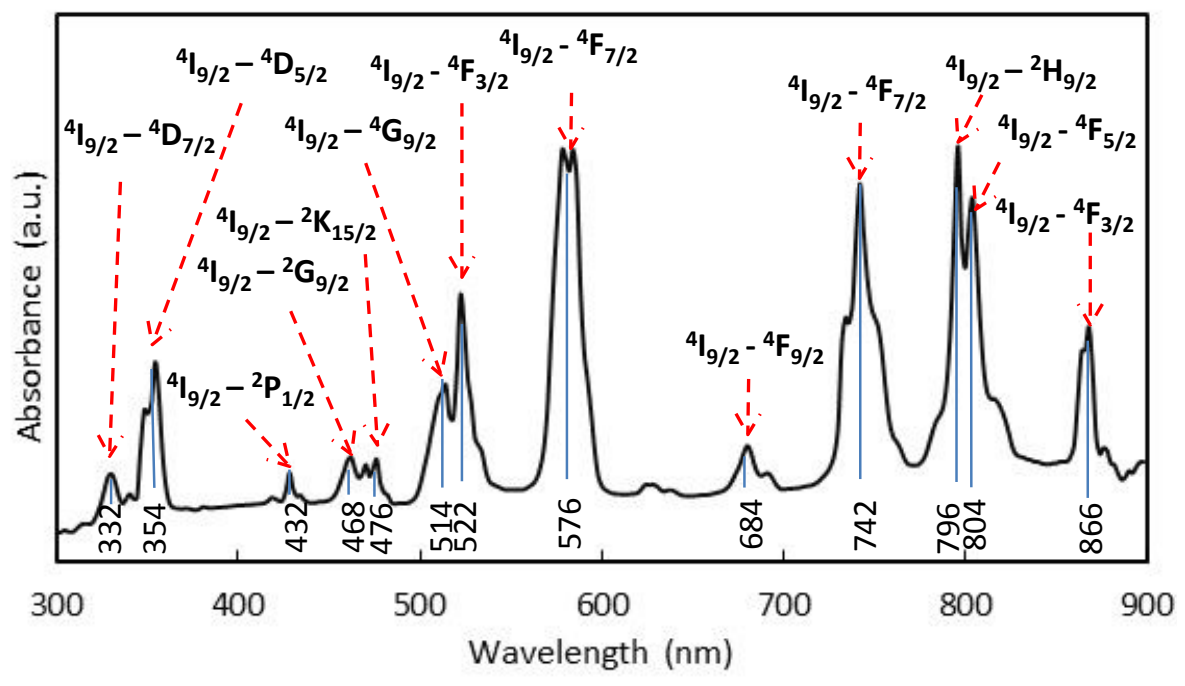

Compound 2-Nd $\mathrm{N}_{2} \mathrm{H}_{5}\left[\left\{\mathrm{Nd}_{2}\left(\mathrm{~N}_{2} \mathrm{H}_{5}\right)\right\}\left(\mathrm{C}_{2} \mathrm{O}_{4}\right)_{4}\right] \cdot 4 \mathrm{H}_{2} \mathrm{O}$

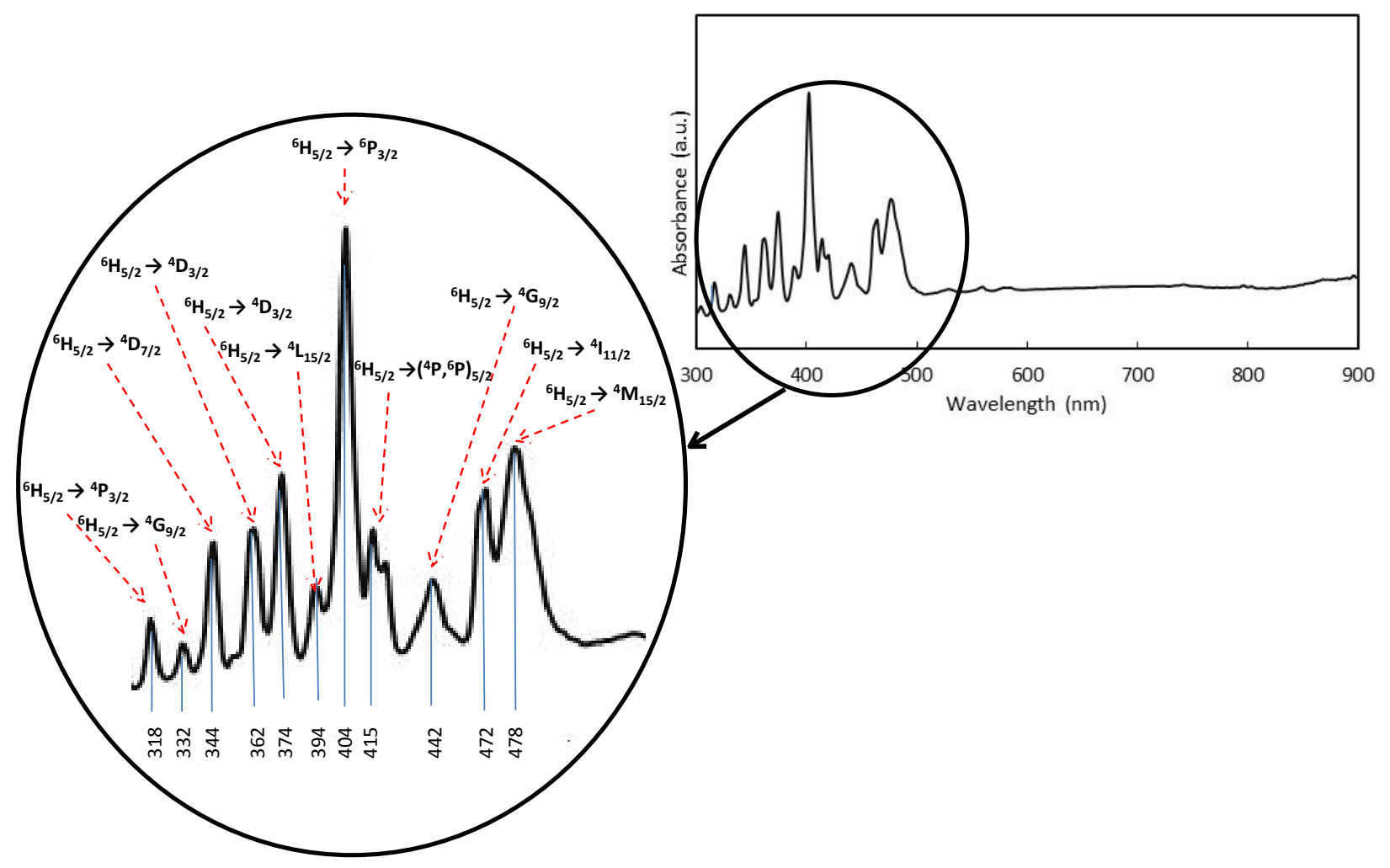

Compound 3-Sm $\left(\mathrm{N}_{2} \mathrm{H}_{5}\right) \mathrm{Sm}\left(\mathrm{C}_{2} \mathrm{O}_{4}\right)_{2} \cdot \mathrm{H}_{2} \mathrm{O}$ 


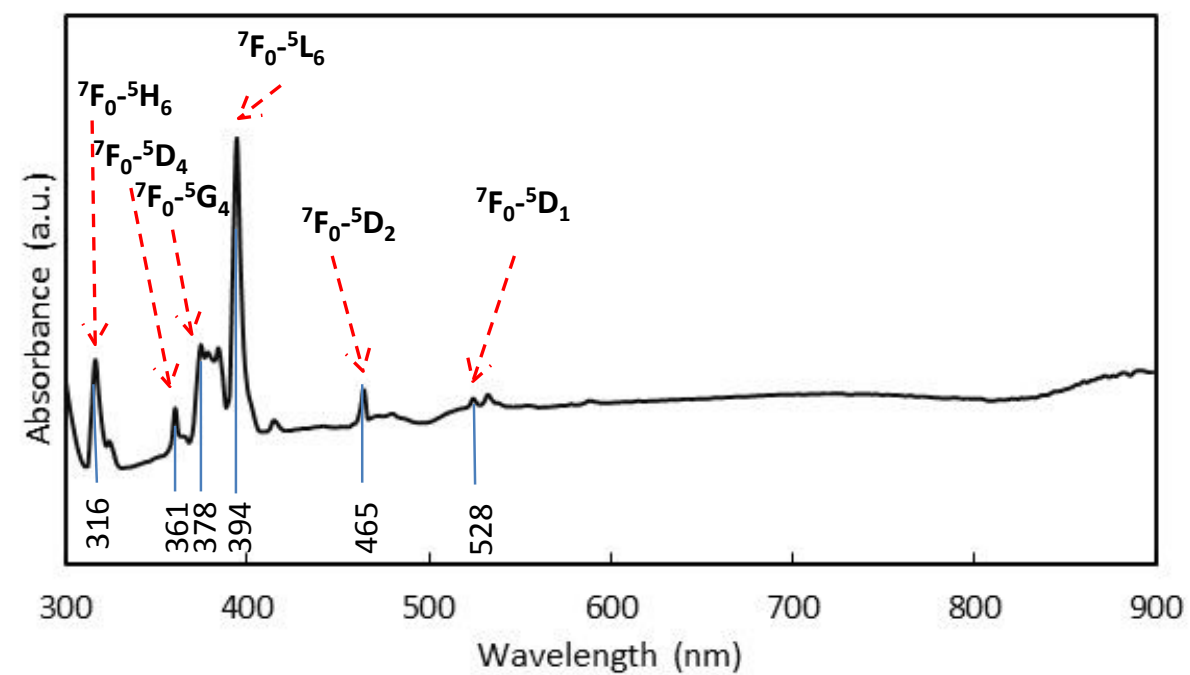

Compound 3-Eu $\left(\mathrm{N}_{2} \mathrm{H}_{5}\right) \mathrm{Eu}\left(\mathrm{C}_{2} \mathrm{O}_{4}\right)_{2} \cdot \mathrm{H}_{2} \mathrm{O}$

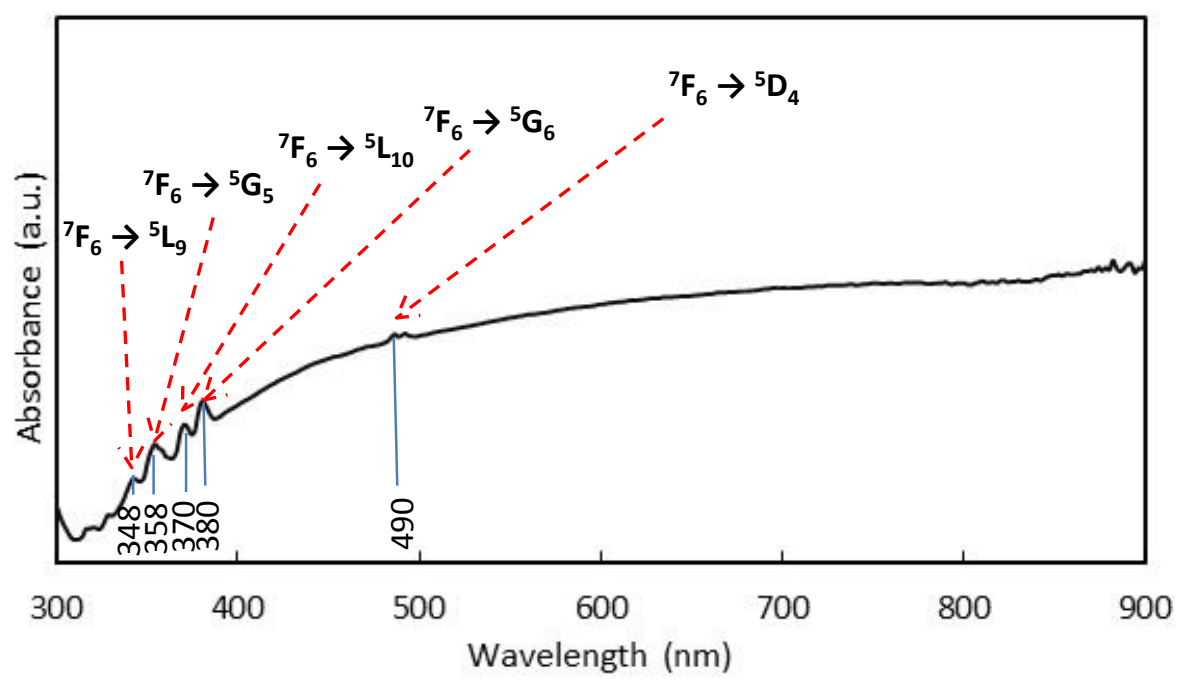

Compound 3-Tb $\left(\mathrm{N}_{2} \mathrm{H}_{5}\right) \mathrm{Tb}\left(\mathrm{C}_{2} \mathrm{O}_{4}\right)_{2} \cdot \mathrm{H}_{2} \mathrm{O}$

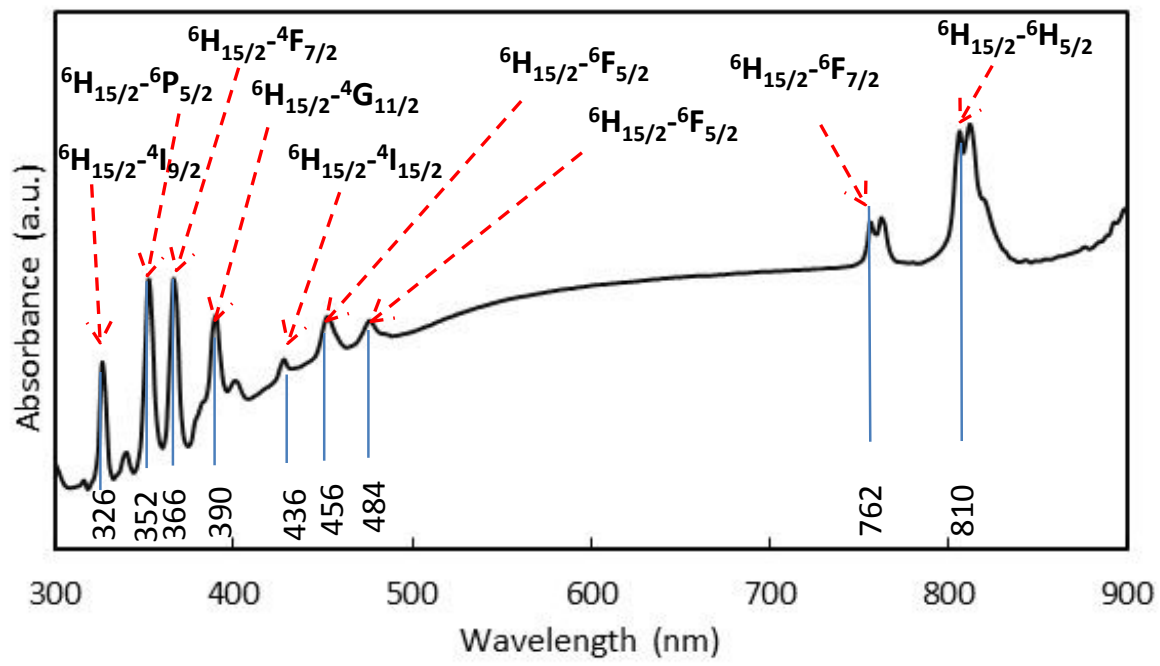

Compound 3-Dy $\left(\mathrm{N}_{2} \mathrm{H}_{5}\right) \mathrm{Dy}\left(\mathrm{C}_{2} \mathrm{O}_{4}\right)_{2} \cdot \mathrm{H}_{2} \mathrm{O}$ 


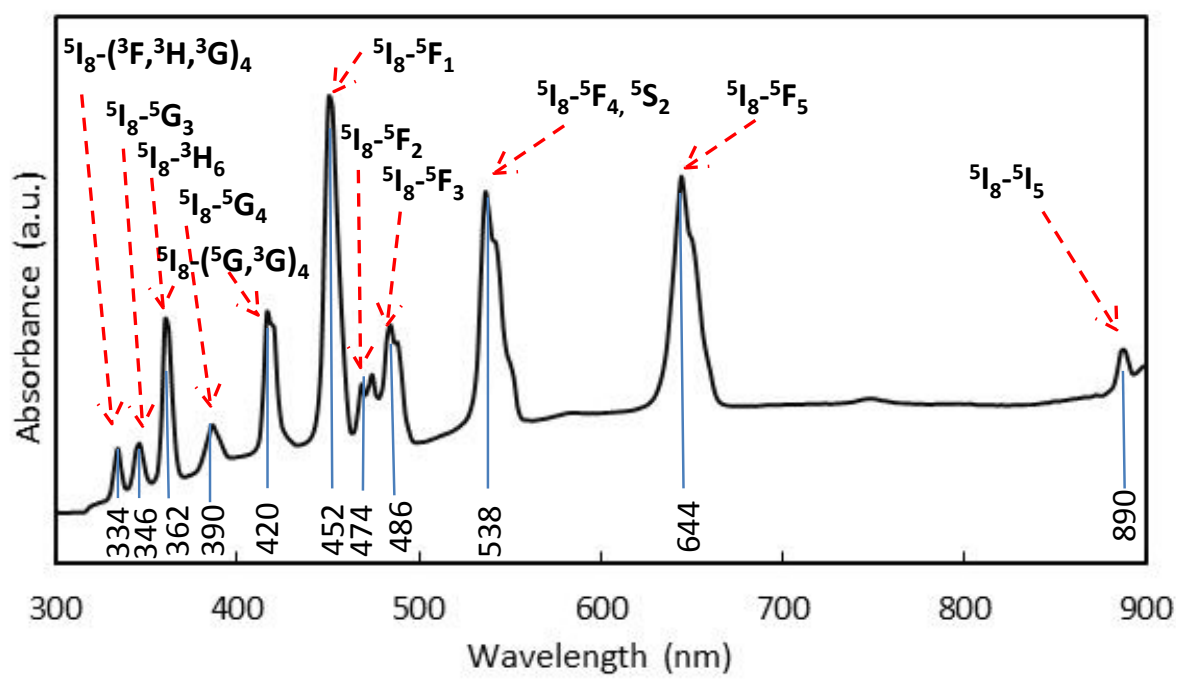

Compound 3-Ho $\left(\mathrm{N}_{2} \mathrm{H}_{5}\right) \mathrm{Ho}\left(\mathrm{C}_{2} \mathrm{O}_{4}\right)_{2} \cdot \mathrm{H}_{2} \mathrm{O}$

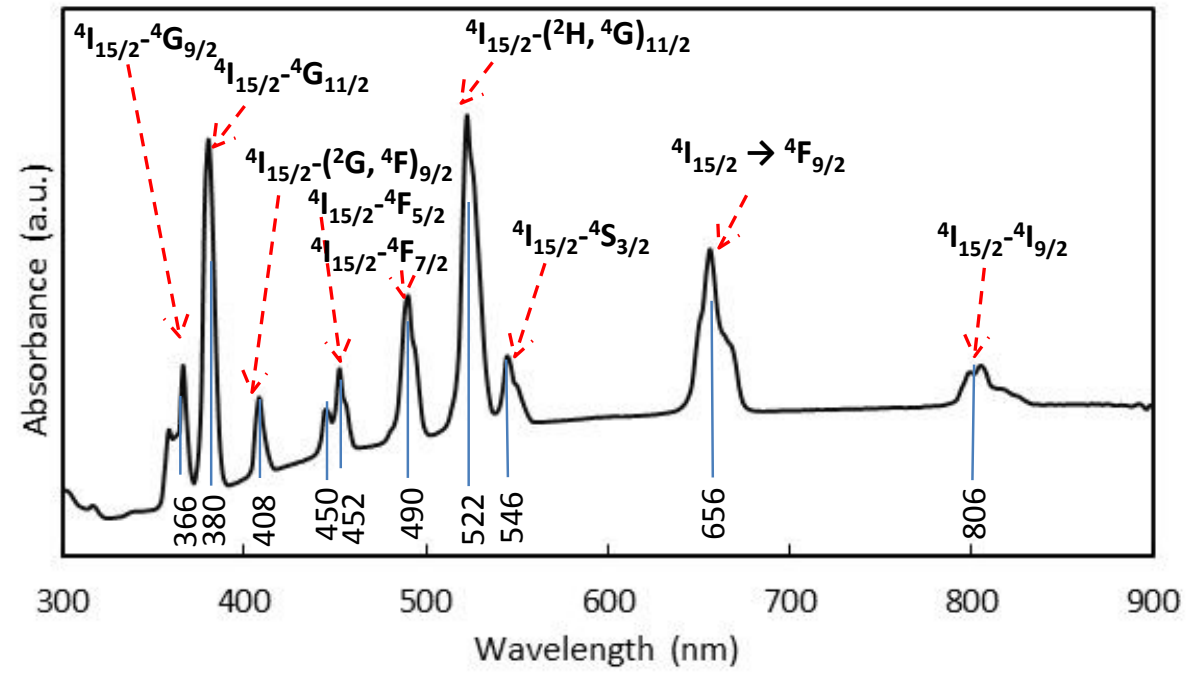

Compound 4-Er $\left[\left\{\operatorname{Er}\left(\mathrm{H}_{2} \mathrm{O}\right)\right\}_{2}\left(\mathrm{C}_{2} \mathrm{O}_{4}\right)_{3}\right] \cdot \mathrm{H}_{2} \mathrm{O}$

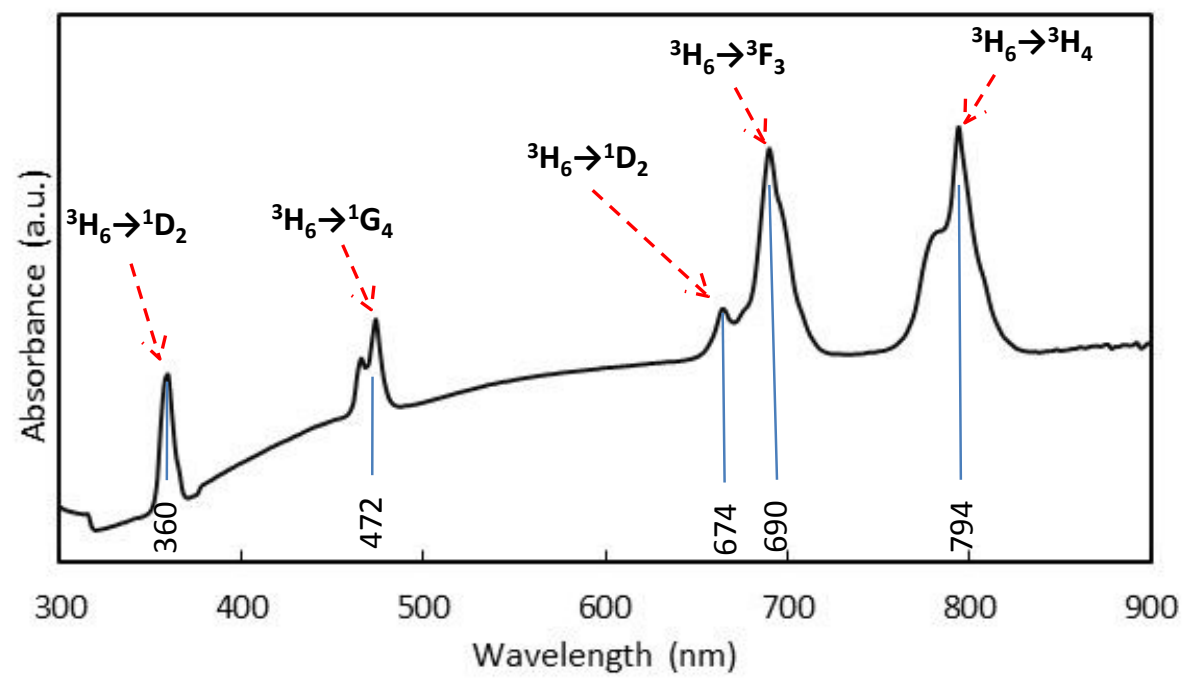

Compound 4-Tm $\left[\left\{\mathrm{Tm}\left(\mathrm{H}_{2} \mathrm{O}\right)\right\}_{2}\left(\mathrm{C}_{2} \mathrm{O}_{4}\right)_{3}\right] \cdot \mathrm{H}_{2} \mathrm{O}$ 